\title{
CMOS Realization of All-Positive Pinched Hysteresis Loops
}

\author{
B. J. Maundy, ${ }^{1}$ A. S. Elwakil, ${ }^{2,3}$ and C. Psychalinos ${ }^{4}$ \\ ${ }^{1}$ Department of Electrical and Computer Engineering, University of Calgary, Calgary, AB, Canada T2N 1N4 \\ ${ }^{2}$ Department of Electrical and Computer Engineering, University of Sharjah, Sharjah, UAE \\ ${ }^{3}$ Nanoelectronics Integrated Systems Center (NISC), Nile University, Giza 12588, Egypt \\ ${ }^{4}$ Physics Department, Electronics Laboratory, University of Patras, Rio, 26504 Patras, Greece
}

Correspondence should be addressed to B. J. Maundy; bmaundy@ucalgary.ca

Received 21 March 2017; Accepted 20 June 2017; Published 6 August 2017

Academic Editor: Jesus M. Munoz-Pacheco

Copyright (C) 2017 B. J. Maundy et al. This is an open access article distributed under the Creative Commons Attribution License, which permits unrestricted use, distribution, and reproduction in any medium, provided the original work is properly cited.

Two novel nonlinear circuits that exhibit an all-positive pinched hysteresis loop are proposed. These circuits employ two NMOS transistors, one of which operates in its triode region, in addition to two first-order filter sections. We show the equivalency to a charge-controlled resistance (memristance) in a decremental state via detailed analysis. Simulation and experimental results verify the proposed theory.

\section{Introduction}

Pinched hysteresis was proposed to be a signature of memristive devices $[1,2]$, yet it can also be observed in several other nonlinear devices such as nonlinear inductors (capacitors) with quadratic-type current (voltage) dependence [3]. Finding a general model for pinched hysteresis behavior was attempted in [4] for specific devices labeled as memristors [5]. In [6] and from a simplified mathematical point of view, the following model was proposed and shown to exhibit a pinched hysteresis behavior which can fit both chargecontrolled and flux-controlled memristance definitions:

$$
y(t)=x(t) \times\left(a+\frac{1}{T} \int_{0}^{t} x(\tau) d \tau\right),
$$

where if $y(t)=v(t)$ and $x(t)=i(t)$, the chargecontrolled memristance is obtained, while for the alternative setting $y(t)=i(t)$ and $x(t)=v(t)$, the flux-controlled memristance is obtained. In (1), the constants $a$ and $T$ are scaling and integration time constants, respectively. Note that circuit realization of this model for the purpose of emulating its pinched hysteresis behavior in non-solid-state devices requires a multiplier block, an integrator block, and an adder [6]. Several other emulator circuits have recently been proposed in the literature [7-13]. It is important to note that (1) is nonlinear due to the multiplication term and that pinched hysteresis cannot appear in a linear system. It is also possible to include other forms of nonlinearity that apply to the shaping of the loop as a result of shaping the applied excitation. This means replacing $x(t)$ in (1) more generally with $f(x(t))$.

Pinched hysteresis loop is generally observed as a result of applying a bipolar sinusoidal voltage or current excitation signal and is thus symmetrical around the origin. Nonsymmetrical loops can also be obtained when the pinch point is shifted away from the origin. However, an all-positive pinched loop, to the best of our knowledge, has not been demonstrated before. It is the purpose of this work to introduce two simple circuits where this behavior is observed. We rely on the inherent nonlinearity of a MOS transistor to perform the multiplication operation required by (1) in order to obtain a charge-controlled memristance. Recall that a MOS transistor current-voltage relation can be described by

$$
i_{\mathrm{ds}}=k\left[\left(v_{\mathrm{gs}}-V_{\mathrm{T}}\right) v_{\mathrm{ds}}-\frac{v_{\mathrm{ds}}^{2}}{2}\right] \text {, }
$$


where $i_{\mathrm{ds}}$ is the drain-to-source current, $v_{\mathrm{gs}}$ and $v_{\mathrm{ds}}$ are, respectively, the gate-to-source and drain-to-source voltage, $V_{T}$ is the threshold voltage, and $k$ is a constant in $A / V^{2}$ units. It is obvious from (2) that a multiplication operation is inherent through the term $\left(v_{\mathrm{gs}}-V_{T}\right) v_{\mathrm{ds}}$. However, for an NMOS transistor, the current is unidirectional and the condition $v_{\mathrm{gs}}>V_{T}$ is necessary for the transistor to switch on. Therefore, if (2) is successfully reconfigured to implement (1), an all-positive pinched hysteresis loop can be obtained. In the first part of this work, we do not attempt to remove the extra nonlinear term $v_{\mathrm{ds}}^{2} / 2$ and therefore it remains affecting the pinched loop. However, this effect is minimized via proper selection of the design parameters. In a later section of the work, we employ a linearization circuit to remove this quadratic term and hence ensure that only the multiplication nonlinearity term remains. As a result and comparing (2) to (1) assuming $v_{\mathrm{ds}}^{2} / 2$ is minimized or eliminated, it is clear that the mapping $i_{\mathrm{ds}} \rightarrow y(t), v_{\mathrm{ds}} \rightarrow x(t)$, and $K\left(v_{\mathrm{gs}}-\right.$ $\left.V_{T}\right) \rightarrow a+(1 / T) \int_{0}^{t} x(\tau) d \tau$ is necessary. To achieve this, we adopt a frequency-domain approach which also allows independent adjustment of the fixed part and the chargecontrolled part of the memristance. We stress and clarify the role played by the capacitors in the proposed circuits, which is crucial to the understanding of pinched hysteresis behavior in general, as clearly seen in $[17,18]$ for solid-state devices as well. Note that, in reconfiguring (2) to realize (1) via this mapping, we are essentially modifying the MOS transistor transconductance such that it is state-controlled, with the state variable being the terminal voltage of the transconductance $v_{\mathrm{ds}}$. The time constant $T$ necessary in (1) can only be obtained with an embedded capacitance (physical or parasitic) $[17,18]$. Finally, it must be stated that this paper is concerned with the "pinched hysteresis behavior" as a behavior rather than with proposing yet another memristor emulator. The design concept of the circuits under study here is completely new and relies on a frequency-domain approach rather than a time-domain approach. It also shows for the first time that pinched hysteresis can even be unipolar, something not possible with memristors as they are so defined.

This manuscript is organized as follows. Section 2 looks at the proposed circuits and presents the theory behind their operation that leads to pinched hysteresis behavior. Section 3 computes the memristance of the proposed circuits, with numerical simulations, and presents pinch-off analysis of the proposed circuits. In Section 4, a method to linearize the main transistor in triode is presented which removes the extra nonlinear term $v_{\mathrm{ds}}^{2} / 2$ in (2). In Section 5, simulation and experimental results are presented, and finally our conclusions are given in Section 6.

\section{The Proposed Circuits}

Consider the circuits shown in Figure 1 both consisting of opamp $A_{1}$ connected as a buffer and opamp $A_{2}$ along with the NMOS transistor $M_{2}$ as a simple voltage to current converter which converts the voltage of the noninverting terminal of $A_{2}$ into a current $i_{\text {in }}$ through NMOS transistor
$M_{1}$. It is essential for $M_{1}$ to remain in triode, hence acting as a transconductance. Both circuits contain a lossy integrator comprising $R_{1}, C_{1}$, and a DC bias voltage $V_{\mathrm{dc}_{1}}$ required to maintain $M_{1}$ in triode $\left(V_{\mathrm{dc}_{1}}>V_{T}, V_{\mathrm{DS}_{1}}<V_{\mathrm{dc}_{1}}-V_{T}\right)$. The difference between the two circuits can be seen in their highpass filter sections. In the case of Figure 1(a), it consists of $R_{2}, C_{2}$ with an additional DC source $V_{d c}$ providing a DC voltage to the drain of $M_{1}$. For Figure 1(b), the high-pass filter is made up of $r_{1,2}, C_{2}$ and here the drain-to-source DC biasing of $M_{1}$ comes from $V_{\mathrm{dc}_{1}}$ through $r_{1,2}$ as $\alpha V_{\mathrm{dc}_{1}}$, where $\alpha=r_{1} /\left(r_{1}+r_{2}\right)$. Note that maintaining transistor $M_{1}$ in triode in both circuits requires that $V_{\mathrm{dc}_{1}}-V_{\mathrm{dc}_{2}}>V_{T}$ and $(1-\alpha) V_{\mathrm{dc}_{1}}>V_{T}$ for Figures 1(a) and 1(b), respectively.

The lossy integrator of each circuit has a response given in the frequency domain which can be written as

$$
H_{1}(\omega)=\left|H_{1}(\omega)\right| \angle-\theta_{1}=\frac{1}{\sqrt{1+\omega^{2} \tau_{1}^{2}}} \angle \tan ^{-1}\left(\omega \tau_{1}\right),
$$

where $\tau_{1}=R_{1} C_{1}$. Meanwhile, the high-pass filter in Figure 1(a) has a response

$$
\begin{aligned}
H_{2 a}(\omega) & =\left|H_{2 a}(\omega)\right| \angle \theta_{2 a} \\
& =\frac{\omega \tau_{2}}{\sqrt{1+\omega^{2} \tau_{2}^{2}}} \angle\left(90^{\circ}-\tan ^{-1}\left(\omega \tau_{2}\right)\right),
\end{aligned}
$$

with $\tau_{2}=R_{2} C_{2}$, and likewise the response of the high-pass filter in Figure 1(b) is

$$
\begin{aligned}
H_{2 b}(\omega) & =\left|H_{2 b}(\omega)\right| \angle \theta_{2 b} \\
& =\alpha \sqrt{\frac{1+\omega^{2} \tau_{z}^{2}}{1+\omega^{2} \tau_{p}^{2}}} \angle \tan ^{-1}\left(\omega \tau_{z}\right)-\tan ^{-1}\left(\omega \tau_{p}\right),
\end{aligned}
$$

where $\tau_{z}=r_{2} C_{2}$ and $\tau_{p}=\left(r_{1} / / r_{2}\right) C_{2}$. Clearly, both highpass filters provide a leading phase shift by different amounts while DC biasing voltages are allowed to be passed on to the transistor $M_{1}$ to set $V_{\mathrm{DS}}$. For an input voltage of amplitude $A$ and frequency $\omega_{o}$ in the form $v_{\text {in }}(t)=A \sin \left(\omega_{o} t\right)+V_{\mathrm{dc}_{1}}$, it follows that the time dependent $V_{\mathrm{GS}}$ voltage of $M_{1}$ is

$$
V_{\mathrm{GS}_{1}}(t)=A\left|H_{1}\left(\omega_{o}\right)\right| \sin \left(\omega_{o} t-\theta_{1}\right)+V_{\mathrm{dc}_{1}},
$$

while the time dependent $V_{\mathrm{DS}}$ voltages in Figures 1(a) and 1(b) are, respectively,

$$
\begin{aligned}
& V_{\mathrm{DS}_{1}}(t)=A\left|H_{2 a}\left(\omega_{o}\right)\right| \sin \left(\omega_{o} t+\theta_{2 a}\right)+V_{\mathrm{dc}_{2}}, \\
& V_{\mathrm{DS}_{1}}(t)=A\left|H_{2 b}\left(\omega_{o}\right)\right| \sin \left(\omega_{o} t+\theta_{2 b}\right)+\alpha V_{\mathrm{dc}_{1}},
\end{aligned}
$$

where, for (7), $A<V_{\mathrm{dc}_{2}}$ and $A<\alpha V_{\mathrm{dc}_{1}}$, respectively. Substituting (6)-(7) into (2) yields after considerable simplification

$$
\begin{aligned}
i_{\mathrm{in}_{a}}(t)= & -\widehat{A} \cos \left(2 \omega_{o} t-\theta_{1}+\theta_{2 a}\right)+\widehat{B} \sin \left(\omega_{o} t-\theta_{1}\right) \\
& +\widehat{C} \sin \left(\omega_{o} t+\theta_{2 a}\right)+\widehat{D} \cos \left(2 \omega_{o} t+2 \theta_{2 a}\right) \\
& +\widehat{E}+\widehat{I}_{\mathrm{off}},
\end{aligned}
$$


TABLE 1: Summary of the coefficients in (8) and (9) for Figures 1(a) and 1(b).

\begin{tabular}{lcc}
\hline Coefficient & Figure 1(a) & Figure 1(b) \\
\hline$\widehat{A}$ & $\frac{1}{2} k A^{2}\left|H_{1}\left(\omega_{o}\right)\right|\left|H_{2 a}\left(\omega_{o}\right)\right|$ & $\frac{1}{2} k A^{2}\left|H_{1}\left(\omega_{o}\right)\right|\left|H_{2 b}\left(\omega_{o}\right)\right|$ \\
$\widehat{B}$ & $k A\left|H_{1}\left(\omega_{o}\right)\right| V_{\mathrm{dc}_{2}}$ & $\alpha k A\left|H_{1}\left(\omega_{o}\right)\right| V_{\mathrm{dc}_{1}}$ \\
$\widehat{C}$ & $k A\left|H_{2 a}\left(\omega_{o}\right)\right|\left(V_{\mathrm{dc}_{1}}-V_{\mathrm{dc}_{2}}-V_{T}\right)$ & $k A\left|H_{2 b}\left(\omega_{o}\right)\right|\left[(1-\alpha) V_{\mathrm{dc}_{1}}-V_{T}\right]$ \\
$\widehat{D}$ & $\frac{1}{4} k A^{2}\left|H_{2 a}\left(\omega_{o}\right)\right|^{2}$ & $\frac{1}{4} k A^{2}\left|H_{2 b}\left(\omega_{o}\right)\right|^{2}$ \\
$\widehat{E}$ & $\widehat{A} \cos \left(\theta_{1}+\theta_{2 a}\right)-\widehat{D}$ & $\widehat{A} \cos \left(\theta_{1}+\theta_{2 b}\right)-\widehat{D}$ \\
$\widehat{I}_{\text {off }}$ & $k\left[\left(V_{\mathrm{dc}_{1}}-V_{T}\right) V_{\mathrm{dc}_{2}}-\frac{1}{2} V_{\mathrm{dc}_{2}}^{2}\right]$ & $\alpha k\left[\left(1-\frac{\alpha}{2}\right) V_{\mathrm{dc}_{1}}-V_{T}\right] V_{\mathrm{dc}_{1}}$ \\
\hline
\end{tabular}

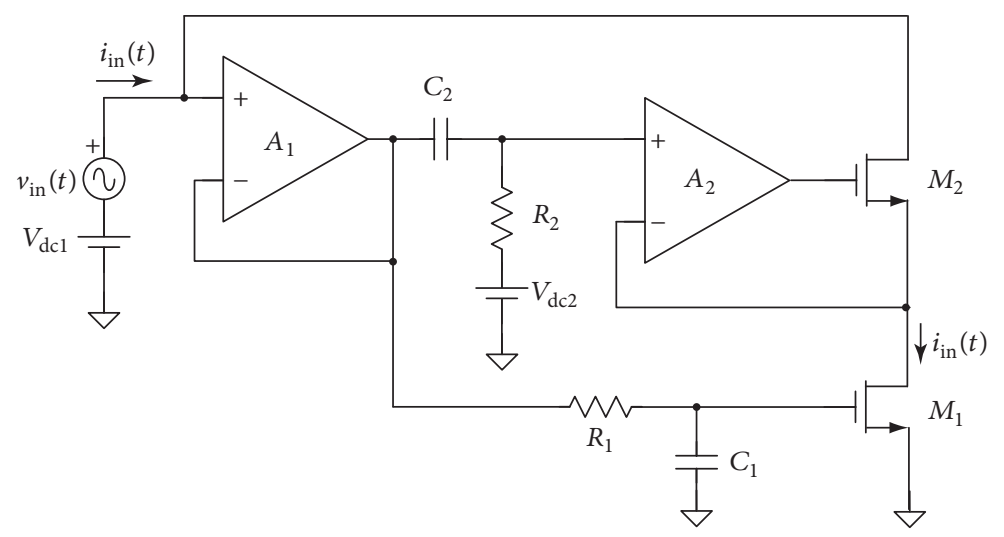

(a)

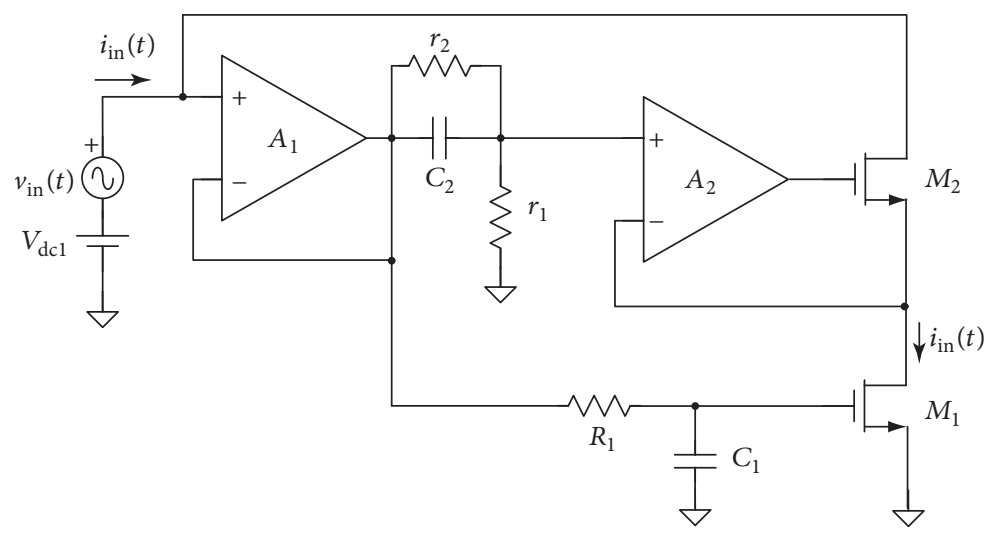

(b)

FIGURE 1: The proposed nonlinear circuits with unipolar pinched hysteresis loop.

for Figure 1(a), and

$$
\begin{aligned}
i_{\text {in }_{b}}(t)= & -\widehat{A} \cos \left(2 \omega_{o} t-\theta_{1}+\theta_{2 b}\right)+\widehat{B} \sin \left(\omega_{o} t-\theta_{1}\right) \\
& +\widehat{C} \sin \left(\omega_{o} t+\theta_{2 b}\right)+\widehat{D} \cos \left(2 \omega_{o} t+2 \theta_{2 b}\right) \\
& +\widehat{E}+\widehat{I}_{\text {off }},
\end{aligned}
$$

for Figure 1(b). The coefficients of the $\sin (\cdot)$ and $\cos (\cdot)$ terms in (8) and (9) are given in Table 1 . Note that, with the exception of $\widehat{I}_{\text {off }}$, all coefficients in Table 1 are frequency dependent. This implies that it is necessary to choose proper values for time constants in order to observe the hysteresis behavior.
Close inspection of (8) under the assumption that $\theta_{2 a} \cong$ $\pi / 2-\theta_{1}$ shows that it can be rewritten in the dimensionless form

$$
\begin{aligned}
y(t)+y_{\mathrm{off}} & \\
= & x(t)\left|H_{1}(\omega)\right|\left[1-\omega\left|H_{2 a}(\omega)\right| \int_{0}^{t} x(\tau) d \tau\right] \\
& +\frac{\left|H_{2 a}(\omega)\right|}{\omega} \dot{x}(t)-\frac{\left|H_{2 a}\left(\omega_{o}\right)\right|^{2}}{2 \omega^{2}}[\dot{x}(t)]^{2}+x_{\mathrm{off}},
\end{aligned}
$$

where $x(t)=\sin \left(\omega t-\theta_{1}\right)$ represents an already phase shifted input signal. The term in the square brackets is clearly similar to (1) while additional $\dot{x}(t)$ and $\dot{x}^{2}(t)$ terms outside the 
TABLE 2: Summary of several analog circuits with pinched hysteresis and actual analog memristor emulators to date and their designs. MB: multiplier block; OPA: operational amplifier; CCII: second-generation current conveyors; AH: additional hardware, which may be in the form of buffers, multiplexers, diodes, inverters, switches, and so forth; I/D: incremental/decremental memristance emulation. The number (\#) of transistors refers to the discrete number of external transistors. *An OTA was used here in place of an OPA.

\begin{tabular}{lccccccc}
\hline Reference [ ] & MB & OPAs & CCIIs & \# of trans. & AH & Quadrant & I/D \\
\hline$[6]$ & 1 & - & 3 & - & Yes & 2 & Both \\
{$[7]$} & - & $1^{*}$ & 2 & - & No & 2 & I \\
{$[8]$} & 1 & - & 2 & - & No & 2 & I \\
{$[9]$} & - & 2 & 1 & 2 & No & 2 & D \\
{$[10]$} & 1 & 5 & - & 10 & Yes & 2 & Both \\
{$[11]$} & - & 2 & 1 & 1 & No & 2 & I \\
{$[12]$} & - & - & 3 & 1 & No & 2 & I \\
{$[13]$} & 1 & 2 & - & - & Yes & 2 & D \\
{$[14]$} & 1 & - & 2 & - & Yes & 2 & I \\
{$[15]$} & - & $1^{*}$ & 1 & 2 & No & 2 & Both \\
{$[16]$} & - & - & 4 & - & Yes & 2 & NA \\
This work & - & 2 & - & 2 & No & 1 & D \\
\hline
\end{tabular}

brackets are unwanted and will result in a nonsymmetrical loop. Note that the offset terms $y_{\text {off }}$ and $x_{\text {off }}$ present are a result of the input being DC level shifted. However, using (10) and assuming that $\omega$ is sufficiently large such that the second and third terms are negligible and in addition translating the origin to $\left(x_{0}, y_{0}\right)=\left(x_{\text {off }}, y_{\text {off }}\right)$, we obtain

$$
\begin{aligned}
y(t) & \\
= & x(t) \\
& \times\left[\left|H_{1}(\omega)\right|-\omega\left|H_{2 a}(\omega)\right|\left|H_{1}(\omega)\right| \int_{0}^{t} x(\tau) d \tau\right],
\end{aligned}
$$

which compared to (1) has the slightly modified form $y(t)=$ $x(t) \times\left(a+(a b / T) \int_{0}^{t} x(\tau) d \tau\right)$, where $a=\left|H_{1}(\omega)\right|$ and $b=$ $-\left|H_{2 a}(\omega)\right|$.

Finally, in comparison with other circuits which also exhibit pinched hysteresis behavior and some of which are labeled as analog memristor emulators as shown in Table 2, about half of them use discrete multiplier blocks which are inefficient. By far, the vast majority use second-generation current conveyors with the only commercial one being the AD844, and several use additional hardware in the form of buffers, multiplexers, diodes, and switches. If the total component count is used as a figure of merit, then the proposed circuits of Figure 1 have the lowest count with their main drawback being operation in one quadrant. Note that even though our proposed circuits are listed among those identified as "memristors" or "memristor emulators," we refrain from labeling our proposed circuits as "memristor emulators" and simply label them as among circuits having pinched hysteresis behavior.

\section{Charge-Controlled Resistance (Memristance) Calculation}

Using (11) and setting $x(t)=i(t) / I_{\text {ref }}, y(t)=v(t) / I_{\text {ref }} R_{s}$, where $I_{\text {ref }}$ is an arbitrary reference current and $R_{s}$ is an arbitrary scaling resistor, the memristance value for Figure 1(a) can be obtained as

$$
R_{m_{a}}=R_{s}\left|H_{1}(\omega)\right|\left[1-\frac{\omega\left|H_{2 a}(\omega)\right|}{I_{\mathrm{ref}}} q(t)\right],
$$

where $q(t)$ is the electrical charge. Note that since the input signal has a fixed $\omega=1 / T$, we can rewrite this memristance as

$$
R_{m_{a}}=R_{s}\left|H_{1}\left(\frac{1}{T}\right)\right|\left[1-\frac{1}{T} \frac{\left|H_{2 a}(1 / T)\right|}{I_{\mathrm{ref}}} q(t)\right],
$$

which has a fixed resistive part equal to $R_{s}\left|H_{1}(1 / T)\right|$ and a charge-controlled part equal to $R_{s}\left|H_{1}(1 / T)\right|\left|H_{2 a}(1 / T)\right|$. It is thus clear that while the transfer function $H_{1}$ controls the magnitude of both parts, $H_{2 a}$ can change the magnitude of the charge-controlled part alone. Note that this memristance is decremental [6]. Furthermore, note that, for sufficiently high frequency such that $\omega \gg 1 / \tau_{1},\left|H_{1}(\omega)\right| \approx 1 / \omega \tau_{1}$ and it follows that the fixed part of the realized memristance is approximately $R_{s}\left(T / \tau_{1}\right)$. However, for sufficiently high frequency such that $\omega \gg 1 / \tau_{2}$, we also note that $\left|H_{2 a}(\omega)\right| \approx 1$ and therefore the realized memristance can be approximated as

$$
R_{m a} \approx R_{s}\left(\frac{T}{\tau_{1}}\right)\left[1-\frac{R_{s}}{T \times 1 V} q(t)\right] .
$$

We can further express the electrical charge as $q(t)=C_{1} v_{C_{1}}(t)$ since the only capacitor in the circuit in this case capable of 


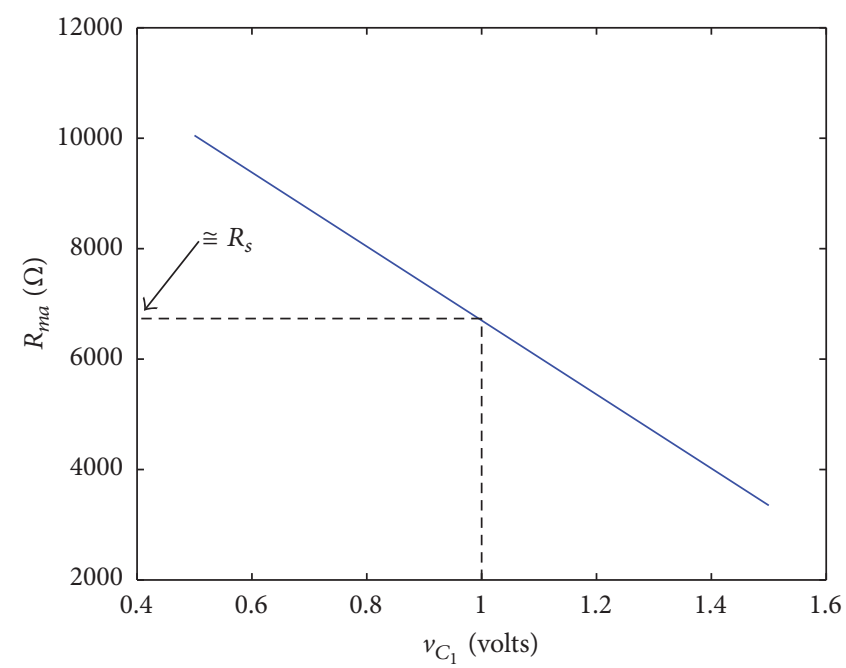

(a)

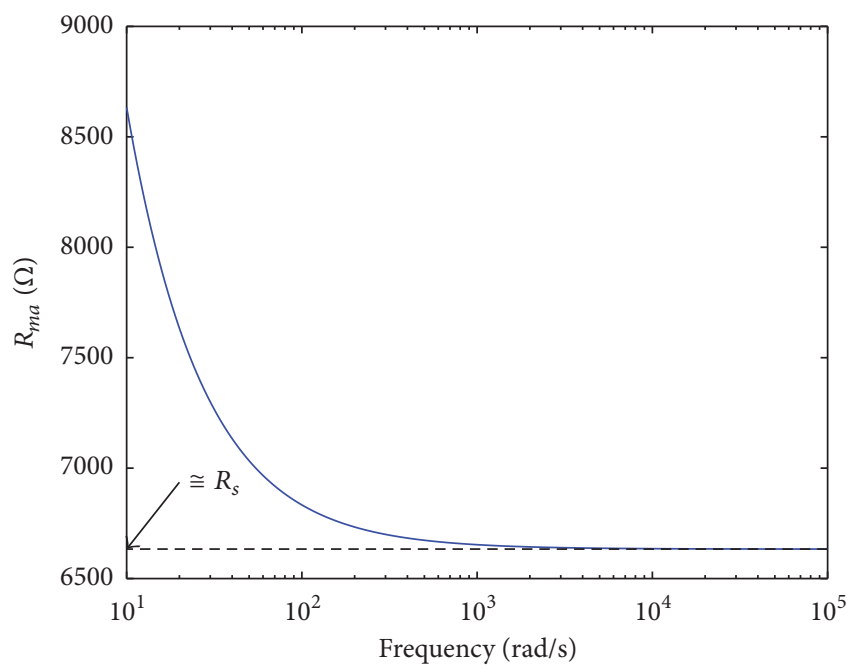

(b)

Figure 2: (a) Plot of memristance $R_{m a}$ versus capacitor voltage $v_{C_{1 n}}=v_{C_{1}} / 1 V$ when $m=v_{C 1 n} / 100, R_{s}=6.7 \mathrm{k} \Omega, R_{\text {off }_{a}}=12.67 \mathrm{k} \Omega$, and $\omega \gg 1 / \tau_{1}$. (b) Plot of $R_{m a}$ versus frequency when $C_{1}=10 \mu \mathrm{F}$.

holding a charge is $C_{1}$ following the fact that $\left|H_{2 a}(\omega)\right| \approx 1$. Accordingly,

$$
\begin{aligned}
R_{m a} & \approx\left(\frac{R_{s} T}{R_{1} C_{1}}\right)\left[1-\frac{R_{s}}{T} C_{1} v_{C_{1 n}}(t)\right] \\
R_{m a} & =\frac{R_{s}^{2}}{R_{1}}\left(\frac{T}{R_{s} C_{1}}-v_{C_{1 n}}(t)\right),
\end{aligned}
$$

where $v_{C_{1 n}}(t)$ is the normalized (by $1 V$ ) voltage across $C_{1}$. In a final step, we may freely express the period $T$ of the applied signal as a ratio of $R_{s} C_{1}$ (i.e., $T=m R_{s} C_{1}$ ) leading to the simplified expression

$$
R_{m a} \approx \frac{R_{s}^{2}}{R_{1}}\left(m-v_{C_{1 n}}(t)\right)=R_{s}\left(m-v_{C_{1 n}}(t)\right)
$$

if we select the arbitrary reference resistance as $R_{s}=R_{1}$. Note that, for the condition $\omega \gg 1 / \tau_{1}$ to be satisfied, it follows that $m \ll 1$.

The expression in (16) is significantly important for two aspects:

(i) It shows that although the circuit has an all-positive input resistance, theoretically and according to (16), the memristance is not always positive. However, it remains positive because the origin has been already shifted to $\left(x_{0}, y_{0}\right)=\left(x_{\text {off }}, y_{\text {off }}\right)$. With reference back to the origin $\left(x_{0}, y_{0}\right)=(0,0),(16)$ then becomes

$$
R_{m a} \approx R_{s}\left(m-v_{\mathrm{Cln}}(t)\right)+R_{\mathrm{off}_{a}},
$$

where $R_{\mathrm{off}_{a}}=V_{\mathrm{dc}_{1}} / V_{\mathrm{dc}_{2}} / k\left[\left(2-V_{\mathrm{dc}_{2}} / V_{\mathrm{dc}_{1}}\right) V_{\mathrm{dc}_{1}}-\right.$ $\left.V_{T}\right]$. Figures 2(a) and 2(b) show the variation of the memristance versus the capacitor voltage $v_{C_{1}}$ and versus frequency $\omega$, respectively. In Figure 2(a), for the values chosen: $m=v_{C_{1}} / 100, V_{\mathrm{dc}_{1}}=1 \mathrm{~V}, V_{\mathrm{dc}_{2}}=$
$0.4 \mathrm{~V}, V_{T}=0.35 \mathrm{~V}$, and $k=0.15 \mathrm{~mA} / \mathrm{V}^{2}$, we obtain $R_{\text {off }}=12.67 \mathrm{k} \Omega$. Correspondingly, selecting a suitable reference current such as $I_{\text {ref }}=0.15 \mathrm{~mA}$ leads to a nominal value for $R_{s}$ of $6.7 \mathrm{k} \Omega$ (i.e., $I_{\text {ref }} R_{s} \approx$ $1 \mathrm{~V}$ ). For Figure 2(b), we fixed $v_{C_{1}}=1 \mathrm{~V}$ and $C_{1}=10 \mu \mathrm{F}$ and show the decremental nature of the memristance whereas the frequency increases when $R_{m a}$ asymptotically approaches $R_{s}$.

(ii) It highlights the significance and necessity of the existence of a capacitor in order to hold the charge. In this circuit, this capacitor is $C_{1}$; however, in solidstate devices, this capacitor may well be a parasitic capacitor or equivalent of parasitic capacitances as observed in $[17,18]$. It thus appears to the authors that it is not possible to isolate the appearance of pinched hysteresis loops from the existence of a capacitive effect.

In a similar manner, the memristance of the circuit in Figure 1(b) can be obtained as

$$
R_{m_{b}}=R_{s}\left|H_{1}\left(\frac{1}{T}\right)\right|\left[\alpha-\frac{1}{T} \frac{\left|H_{2 b}(1 / T)\right|}{I_{\text {ref }}} q(t)\right],
$$

which unlike (13) has a fixed resistive part equal to $\alpha R_{s}\left|H_{1}(1 / T)\right|$ but an identical charge-controlled part. Noting that $\left|H_{2 b}(1 / T)\right|=\alpha$ for $1 / T \gg 1 / \tau_{1}$ and making the same assumptions as before, a generalized expression for $R_{m b}$ can be given as

$$
\begin{aligned}
& R_{m b} \approx \alpha \frac{R_{s}^{2}}{R_{1}}\left(m-v_{C_{1 n}}(t)\right)+R_{\text {off }_{b}} \\
& R_{m b}=\alpha R_{s}\left(m-v_{C_{1 n}}(t)\right)+R_{\text {off }_{b}},
\end{aligned}
$$

where $R_{\text {off }_{b}}=1 / \alpha k\left[(2-\alpha) V_{\mathrm{dc}_{1}}-V_{T}\right]$. 


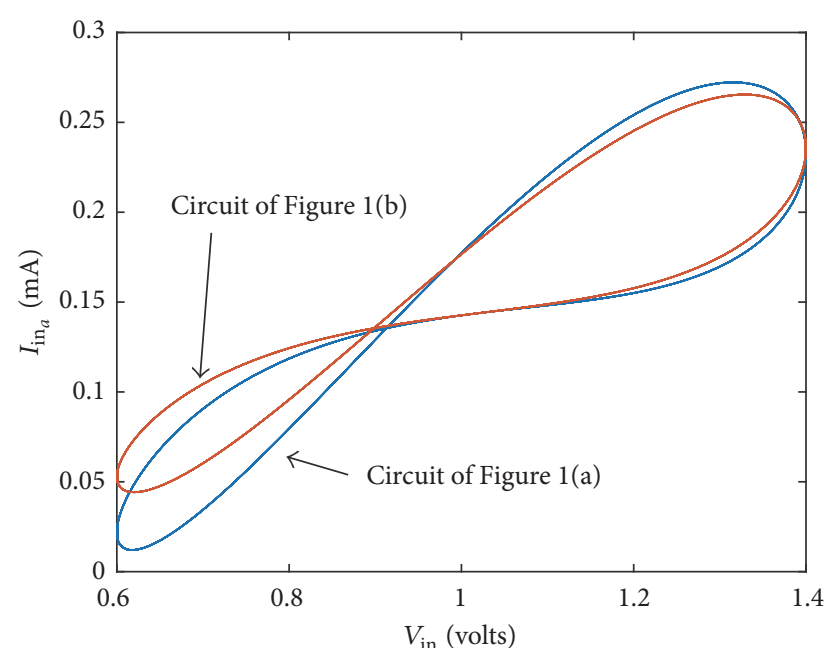

(a)

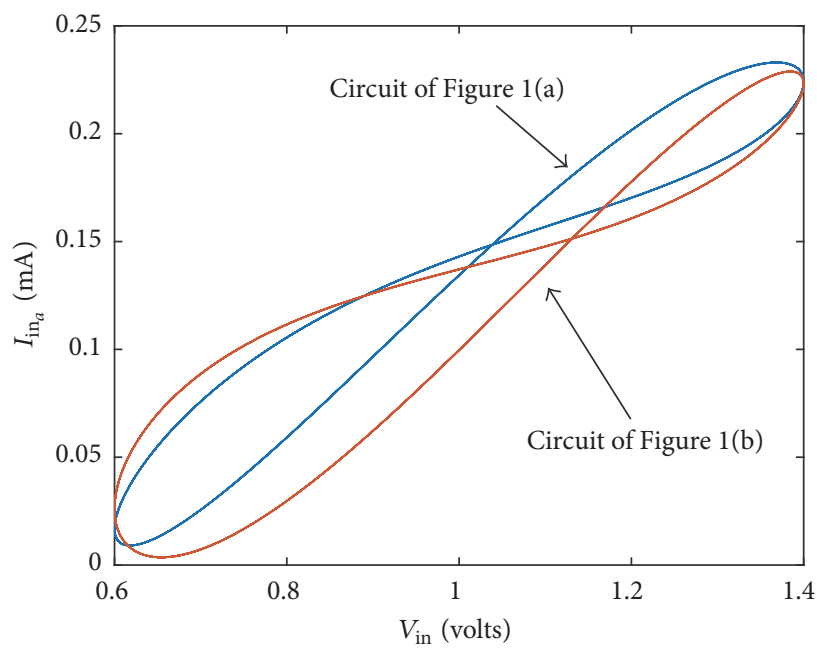

(c)

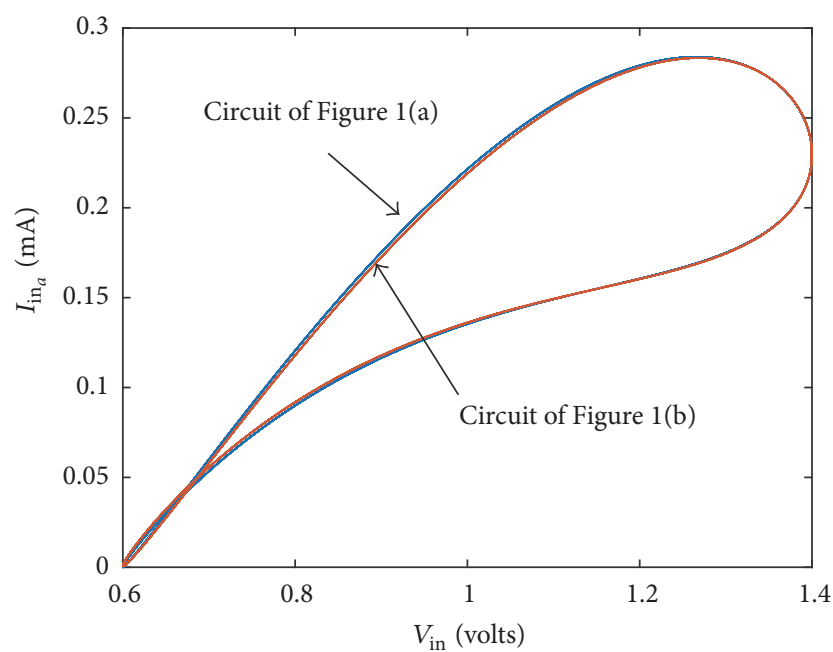

(b)

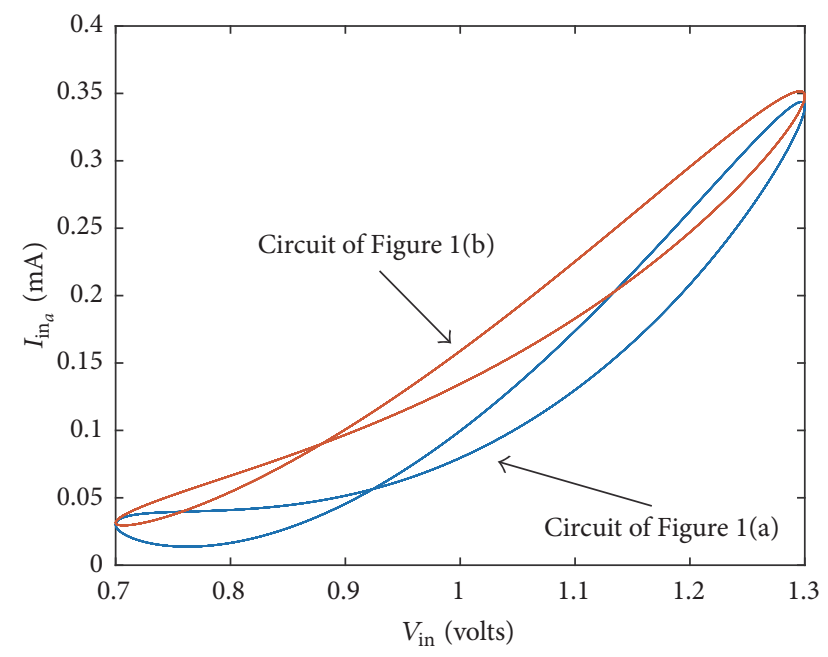

(d)

FIGURE 3: Matlab simulation of the $I-V$ characteristics of Figures 1(a) and 1(b) as given by (8) and (9), respectively. (a) $\tau_{2}=\tau_{1}=\tau_{z}, \tau_{p}=\alpha \tau_{z}$ with $\alpha=0.4$. (b) $\tau_{2}=10 \tau_{1}=\tau_{z}, \tau_{p}=\alpha \tau_{z}$ with $\alpha=0.4$. (c) $\tau_{2}=\tau_{1}=\tau_{z}, \tau_{p}=\alpha \tau_{z}$ with $\alpha=0.286$ and $V_{\mathrm{dc}_{2}}=0.3 \mathrm{~V}$. (d) $\tau_{2}=\tau_{1}=0.1 \tau_{z}$, $\tau_{p}=\alpha \tau_{z}$ with $\alpha=0.33, V_{\mathrm{dc}_{2}}=0.3 \mathrm{~V}$, and $A=0.5 \mathrm{~V}$ and $A=0.3 \mathrm{~V}$ for Figures $1(\mathrm{a})$ and $1(\mathrm{~b})$, respectively.

3.1. Numerical Simulations. Sample Matlab plots of (8) and (9) are shown in Figures 3(a)-3(c) to normalized values of $k=$ $1 \mathrm{~mA} / \mathrm{V}^{2}, A=0.4 \mathrm{~V}, V_{\mathrm{dc}_{1}}=1 \mathrm{~V}$, and $V_{T}=0.35 \mathrm{~V}$. In the first of the plots shown in Figure $3(\mathrm{a}), \tau_{2}=\tau_{1}=\tau_{z}, \tau_{p}=\alpha \tau_{z}$ with $\alpha=0.4$ and the applied sinusoidal voltage frequency $\omega_{o}>$ $1 / \tau_{1}$ and $V_{\mathrm{dc}_{2}}=0.4 \mathrm{~V}$. Note that having $\alpha=0.4$ is equivalent to setting $V_{\mathrm{dc}_{2}}=\alpha V_{\mathrm{dc}_{1}}$ and that $\theta_{2 b} \approx \theta_{2 a}=\pi / 2-\theta_{1}$. In this figure, we see that neither loop is symmetrical which is attributed to the $\dot{x}(t)$ and $\dot{x}^{2}(t)$ terms (see (10)) and the phase shift term introduced by the lossy integrator. In the second plot, shown in Figure 3(b), $\tau_{2}=10 \tau_{1}=\tau_{z}, \tau_{p}=\alpha \tau_{z}$ with $\alpha=0.4$ and $V_{\mathrm{dc}_{2}}=0.4 \mathrm{~V}$ as before but $\theta_{2 a} \approx \theta_{2 b} \cong 0$. The pinched loops from the two circuits are nearly identical and the upper lobe is far bigger than the lower one. In Figure 3(c), $\tau_{2}=\tau_{1}=\tau_{z}, \tau_{p}=\alpha \tau_{z}$ with $\alpha=0.286, V_{\mathrm{dc}_{2}}=0.3 \mathrm{~V}$, and $\theta_{2 b} \approx \theta_{2 a}=\pi / 2-\theta_{1}$. Clearly, as $\alpha$ or $V_{\mathrm{dc}_{2}}$ decreases, the upper lobe decreases in size and the pinch point increases. Note the reduced value of $A$ for the circuit (Figure 1(a) or (8)). This implies that the circuit of Figure 1(a) must work with reduced input amplitudes compared to the circuit of Figure 1(b), unless $V_{\mathrm{dc}_{1}}$ and $V_{\mathrm{dc}_{2}}$ are adjusted in tandem. This is not the case for the circuit of Figure 1(b) where DC bias voltages are related by $\alpha$ which is fixed for $\omega_{o}>1 / \tau_{1}$.

Finally, in Figure 3(d), $\omega<1 / \tau_{1}$ with $\tau_{2}=\tau_{1}=0.1 \tau_{z}$, $\tau_{p}=\alpha \tau_{z}, \alpha=0.33$, and $V_{\mathrm{dc}}=0.3 \mathrm{~V}$. Under these conditions, $\theta_{2 b} \ll \theta_{2 a}=\pi / 2-\theta_{1}$, and decreased amplitudes must now be used in the circuit of Figure 1(b) or (9) compared to the circuit of Figure 1(a) or (8). Therefore, we select $A=0.5 \mathrm{~V}$ and $A=0.3 \mathrm{~V}$ for the two circuits, respectively, in this case.

3.2. Pinch Point Analysis. The unique form of (8) and (9) allows for a closed-loop solution of the pinch-off point in 


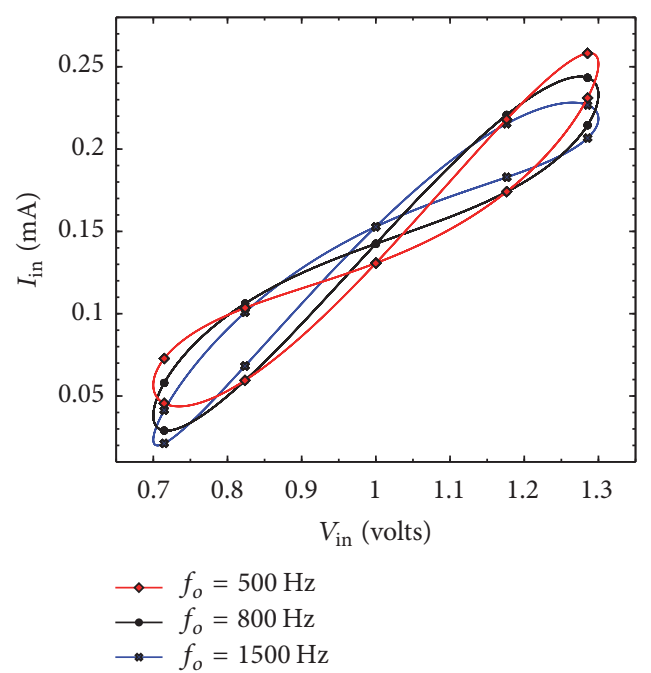

(a)

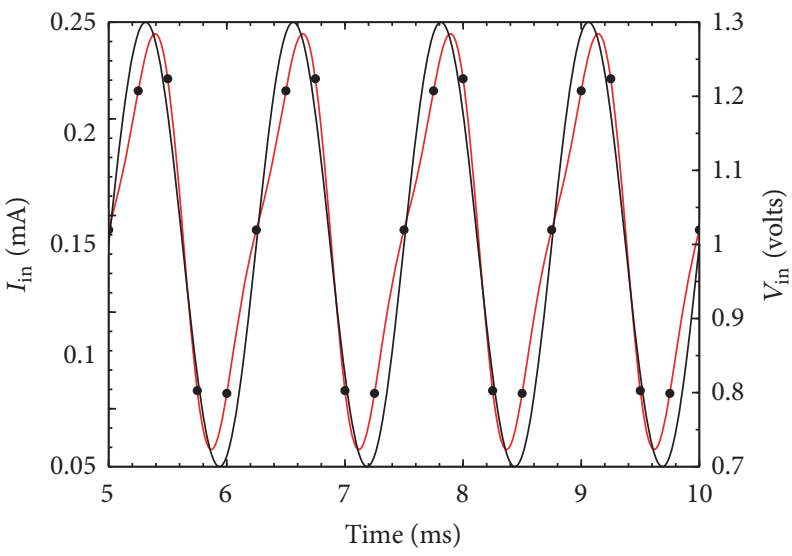

(b)

Figure 4: (a) Matlab simulation of the $I-V$ characteristic of Figure 1 (a) governed by (8) to $A=0.3 \mathrm{~V}, \tau_{2}=\tau_{1}=500 \mu \mathrm{s}, \mathrm{V}_{\mathrm{dc}_{1}}=1 \mathrm{~V}, V_{T}=0.35 \mathrm{~V}$, $k=1 \mathrm{~mA} / \mathrm{V}^{2}$, and $V_{\mathrm{dc}_{2}}=0.325 \mathrm{~V}$. (b) Input voltage $v_{\mathrm{in}}(t)$ and current $i_{\mathrm{in}_{a}}(t)$ plotted as a function of time at a frequency of $\omega=2 \pi \times 800 \mathrm{rad} / \mathrm{s}$.

these circuits. It can be shown after considerable simplification that (8) and (9) have a pinch-off point $\left[\widehat{V}_{\mathrm{in}_{p}}, \widehat{I}_{\mathrm{in}_{p}}\right]$ given by

$$
\begin{aligned}
\widehat{V}_{\mathrm{in}_{p}}= & V_{\mathrm{dc}_{1}}-A \widehat{X}(\Theta) \\
\widehat{I}_{\mathrm{in}_{p}=}= & -\widehat{A} \cos \left[2 \sin ^{-1}(X(\Theta))+\theta_{2 a, 2 b}-\theta_{1}\right] \\
& -\widehat{B} \sin \left[\sin ^{-1}(X(\Theta))-\theta_{1}\right] \\
& -\widehat{C} \sin \left[\sin ^{-1}(X(\Theta))+\theta_{2 a, 2 b}\right] \\
& +\widehat{D} \cos \left[2 \sin ^{-1}(X(\Theta))+2 \theta_{2 a, 2 b}\right]+\widehat{E} \\
& +\widehat{I}_{\mathrm{off}},
\end{aligned}
$$

where

$$
\widehat{X}(\Theta)=\frac{(\widehat{B} / 2) \sin \left(-\theta_{1}\right)+(\widehat{C} / 2) \sin \left(\theta_{2 a, 2 b}\right)}{\widehat{A} \sin \left(\theta_{2 a, 2 b}-\theta_{1}\right)-\widehat{D} \sin \left(2 \theta_{2 a, 2 b}\right)},
$$

and the subscripts $a, b$ refer to (8) and (9), respectively. The frequency dependent nature of (20) makes their analysis difficult; however, several observations can be deduced. First, $\widehat{X}(\Theta)$ can be positive or negative depending on the values of $\theta_{1}, \theta_{2 a}$, and $\theta_{2 b}$ as observed in Figure 3. In addition, $C_{2}$ plays an important role even though it is not the main charge holding or integrating capacitor. In Figure 1(a), its minor role is to block $V_{\mathrm{dc}_{1}}$ as $V_{\mathrm{dc}_{2}}$ is passed, but in both circuits, its main contribution is to add a leading phase shift opposed to the lagging phase shift caused by $C_{1}$. For example, in the circuit of Figure 1(b), in the absence of $C_{2}$, that is, if $C_{2}=0$, then $\sin \left(\theta_{2 b}\right)=\sin \left(2 \theta_{2 b}\right)=0$, because $\theta_{2 b}=0$, and with $\left|H_{2 b}(\omega)\right|=$ $\alpha$, (21) reduces to $\widehat{X}(\Theta)=V_{\mathrm{dc}_{1}} / A$, setting $\widehat{V}_{\mathrm{in}_{p}}=0$. That is, no pinch point will occur.
Secondly, under the assumption $\tau_{2}=\tau_{1}$, we find that $\widehat{V}_{\mathrm{in}_{p}}=V_{\mathrm{dc}_{1}}$ when $\tan \left(\theta_{1}\right)=\widehat{C} / \widehat{B}$ or when $V_{\mathrm{dc}_{2}}=\left(V_{\mathrm{dc}_{1}}-\right.$ $\left.V_{T}\right) / 2$. Under this condition, $M_{1}$ remains in triode so long as $V_{\mathrm{dc}_{1}}>3 V_{T}$ which is easily satisfied. For the general case when $\tau_{2}=\beta \tau_{1}$, implying that $\theta_{2 a} \neq \pi / 2-\theta_{1}$ (unless $\beta=1$ ), the general solution to $\widehat{X}(\Theta)=0$ yields

$$
V_{\mathrm{dc}_{2}}=\frac{\beta}{(\beta+1)} \frac{\left(\tau_{1}^{2} \omega^{2}+1\right)}{\left(\beta \tau_{1}^{2} \omega^{2}+1\right)}\left(V_{\mathrm{dc}_{1}}-V_{T}\right) .
$$

Likewise, the value for $\alpha$ in the circuit of Figure 1(b) that results in $\widehat{V}_{\mathrm{in}_{p}}=V_{\mathrm{dc}_{1}}$ can be expressed as

$$
\alpha=1-\frac{V_{T}}{V_{\mathrm{dc}_{1}}}-\frac{\omega \tau_{1} \csc \left(\theta_{2 b}\right)}{\left(\omega^{2} \tau_{1}^{2}+1\right)} \sqrt{\frac{1+\omega^{2} \tau_{p}^{2}}{1+\omega^{2} \tau_{z}^{2}}} .
$$

In both general cases, (22) and (23) are frequency dependent, the exception being when $\beta=1$ for (22), but both can be minimized for frequency dependance by ensuring that $\omega \tau_{1}>$ $1, \omega \tau_{z}>1$, and $\omega \tau_{p}>1$. Sample plots of the $I-V$ characteristic for the circuit of Figure 1(a) governed by (8) to the conditions $A=0.3 \mathrm{~V}, \tau_{2}=\tau_{1}=500 \mu \mathrm{s}, V_{\mathrm{dc}_{1}}=1 \mathrm{~V}, V_{T}=0.35 \mathrm{~V}, k=$ $1 \mathrm{~mA} / \mathrm{V}^{2}$, and $V_{\mathrm{dc}_{2}}=\left(V_{\mathrm{dc}_{1}}-V_{T}\right) / 2=0.325 \mathrm{~V}$ are shown in Figure 4(a).

Note that because $\beta=1$ and $V_{\mathrm{dc}_{2}}=\left(V_{\mathrm{dc}_{1}}-V_{T}\right) / 2$, $\widehat{V}_{\mathrm{in}_{p}}=V_{\mathrm{dc}_{1}}$ is independent of the input frequency which is verified at the three frequencies $f_{o}=[500,800,1500] \mathrm{Hz}$, as theoretically predicted. In Figure $4(\mathrm{~b}), v_{\mathrm{in}}(t)$ and $i_{\mathrm{in}_{a}}(t)$ are plotted as a function of time. 


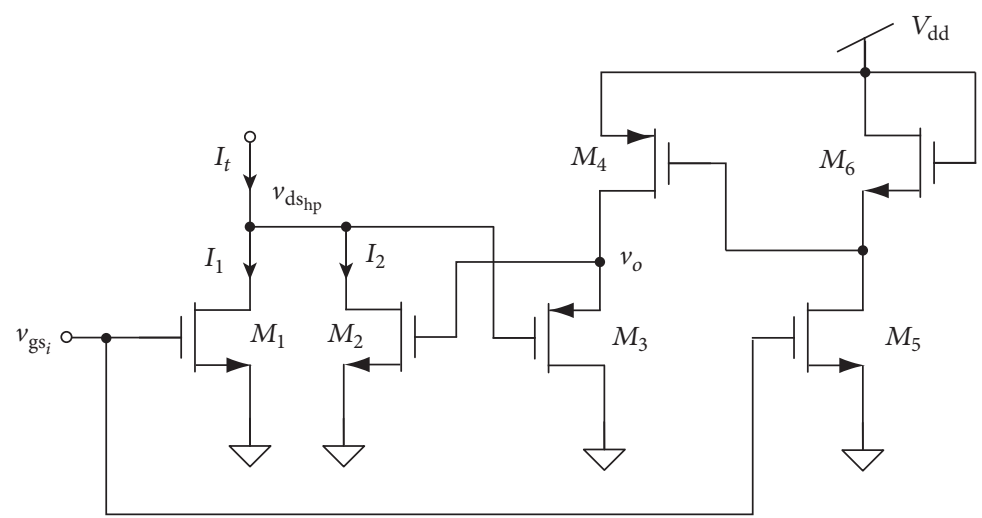

Figure 5: An improved linear resistor obtained by replacing $M_{1}$ in Figure 1 with transistors $M_{1-6}$.

Finally, no pinch point occurs when $\widehat{X}(\Theta)=V_{\mathrm{dc}_{1}} / A$ or $\widehat{X}(\Theta)=-V_{\mathrm{dc}_{1}} / A$ and if $\beta=1$ this happens at the two frequencies $\omega_{1,2}$ :

$$
\begin{aligned}
& \omega_{1}=\frac{1}{\tau_{1}} \sqrt{\frac{2 V_{\mathrm{dc}_{2}}+V_{T}}{3 V_{\mathrm{dc}_{1}}-2 V_{\mathrm{dc}_{2}}-V_{T}}}, \\
& \omega_{2}=\frac{1}{\tau_{1}} \sqrt{\frac{2 V_{\mathrm{dc}_{1}}-2 V_{\mathrm{dc}_{2}}-V_{T}}{V_{\mathrm{dc}_{1}}+2 V_{\mathrm{dc}_{2}}+V_{T}} .}
\end{aligned}
$$

Furthermore, if $V_{\mathrm{dc}_{2}}=(1 / 2)\left(V_{\mathrm{dc}_{1}}-V_{T}\right)$, a unique frequency at which no pinch point exists is

$$
\omega_{1}=\omega_{2}=\frac{1}{\sqrt{2} \tau_{1}} .
$$

In the case of Figure 1(b), no easy closed-loop form of the solution exists, but when $\tau_{z}=\tau_{1}$ the frequencies at which the pinch point occurs can be obtained by numerically solving, for $\omega$, the equation

$$
\gamma_{1} \omega^{4}+\delta_{1} \omega^{2}+2(\alpha-1) V_{\mathrm{dc}_{1}}+V_{T}=0
$$

when $\widehat{X}(\Theta)=V_{\mathrm{dc}_{1}} / A$ or

$$
\begin{aligned}
& \gamma_{2} \omega^{4}+\delta_{2} \omega^{2}+\left(2 \alpha V_{\mathrm{dc}_{1}}+V_{T}\right)\left(\tau_{1}-\tau_{p}\right)+2 V_{\mathrm{dc}_{1}} \tau_{p} \\
& \quad=0,
\end{aligned}
$$

when $\widehat{X}(\Theta)=-V_{\mathrm{dc}_{1}} / A$, where

$$
\begin{aligned}
\gamma_{1}= & \tau_{p}^{3} \tau_{1} V_{\mathrm{dc}_{1}}+\left[(\alpha-1) V_{\mathrm{dc}_{1}}+V_{T}\right] \tau_{p}^{2} \tau_{1}^{2}+\alpha \tau_{p} \tau_{1}^{3} V_{\mathrm{dc}_{1}}, \\
\delta_{1}= & {\left[(\alpha-2) \tau_{p}^{2}+(\alpha+1) \tau_{p} \tau_{1}+(2 \alpha-1) \tau_{1}^{2}\right] V_{\mathrm{dc}_{1}} } \\
& +\left(\tau_{1}^{2}+\tau_{p}^{2}\right) V_{T}, \\
\gamma_{2}= & \tau_{p}^{4} \tau_{1} V_{\mathrm{dc}_{1}}+\left[(2-\alpha) V_{\mathrm{dc}_{1}}-V_{T}\right] \tau_{p}^{3} \tau_{1}^{2} \\
& +\left(V_{T}-V_{\mathrm{dc}_{1}}\right) \tau_{p}^{2} \tau_{1}^{3}+\alpha \tau_{p} \tau_{1}^{4} V_{\mathrm{dc}_{1}}, \\
\delta_{2}= & {\left[(2-\alpha) V_{\mathrm{dc}_{1}}-V_{T}\right] \tau_{p}^{3}+\left(V_{\mathrm{dc}_{1}}+V_{T}\right) \tau_{p}^{2} \tau_{1} } \\
& +\left[(2-\alpha) V_{\mathrm{dc}_{1}}-V_{T}\right] \tau_{p} \tau_{1}^{2} \\
& +\left[(2 \alpha-1) V_{\mathrm{dc}_{1}}+V_{T}\right] \tau_{1}^{3} .
\end{aligned}
$$

\section{Linearization of $M_{1}$}

It is possible to reduce the distortion in $M_{1}$ as a result of the $V_{\mathrm{ds}}^{2} / 2$ term in (2) by a number of techniques. One such technique is bisection of the input range first popularized by [19-21] and exploited by many others. Consider therefore the circuit shown in Figure 5 where $M_{1}$ is replaced by transistors $M_{1-6}$. Voltages $v_{\mathrm{gs}_{i}}$ and $v_{\mathrm{ds}_{h p}}$ form the output voltages of the lossy integrator and high-pass filters, respectively. Transistors $M_{1,2}$ are identical in size as are $M_{3,4}$ and $M_{5,6}$. Transistors $M_{3,4}$ and $M_{5,6}$ are to function as an adder as first proposed by [22] in the use of low voltage multipliers. Under the correct bias conditions, it follows that $v_{o}=v_{\mathrm{gs}_{i}}+v_{\mathrm{ds}_{h p}}$, and then given that both $M_{1}$ and $M_{2}$ have the same $v_{\mathrm{ds}}$ and each drain current is governed by $I_{1}=k\left[\left(v_{\mathrm{gs}_{i}}-V_{T}\right) v_{\mathrm{ds}_{h p}}-v_{\mathrm{ds}_{h p}}^{2} / 2\right]$ and $I_{2}=k\left[\left(v_{o}-V_{T}\right) v_{\mathrm{ds}_{h p}}-v_{\mathrm{ds}_{h p}}^{2} / 2\right]$, the total current $I_{t}$ through $M_{1}$ and $M_{2}$ simplifies to $I_{t}=I_{1}+I_{2}=2 k\left(v_{\mathrm{gs}_{i}}-V_{T}\right) v_{\mathrm{ds}_{h p}}$ or an equivalent resistance of $R_{\mathrm{eq}}=1 / 2 k\left(V_{\mathrm{gs}_{i}}-V_{T}\right)$. Under these conditions, (8) and (9) change to

$$
\begin{aligned}
i_{\mathrm{in}_{a}}(t)= & -\widetilde{A} \cos \left(2 \omega_{o} t-\theta_{1}+\theta_{2 a}\right)+\widetilde{B} \sin \left(\omega_{o} t-\theta_{1}\right) \\
& +\widetilde{C} \sin \left(\omega_{o} t+\theta_{2 a}\right)+\widetilde{A} \cos \left(\theta_{1}+\theta_{2 a}\right)+\widetilde{I}_{\mathrm{off}},
\end{aligned}
$$

and for Figure 1(b)

$$
\begin{aligned}
i_{\mathrm{in}_{b}}(t)= & -\widetilde{A} \cos \left(2 \omega_{o} t-\theta_{1}+\theta_{2 b}\right)+\widetilde{B} \sin \left(\omega_{o} t-\theta_{1}\right) \\
& +\widetilde{C} \sin \left(\omega_{o} t+\theta_{2 b}\right)+\widetilde{A} \cos \left(\theta_{1}+\theta_{2 b}\right)+\widetilde{I}_{\mathrm{off}},
\end{aligned}
$$

where the new coefficients $\widetilde{A}, \widetilde{B}$, and $\widetilde{C}$ are given in Table 3 . Comparing (8)-(9) with (29)-(30) and likewise Tables 1 and 3 , one notices fewer terms for the linearized resistor, with coefficients $\widetilde{A}=\widehat{A}$ and $\widetilde{B}=\widehat{B}$. In addition, both $\widetilde{C}>\widehat{C}$ and 
TABLE 3: Summary of the coefficients of the input current $i_{\text {in }}(t)$ of Figures $1(\mathrm{a})$ and $1(\mathrm{~b})$ in response to an input voltage $v_{\text {in }}(t)$ when $M_{1}$ is replaced by a linearized resistor such as the one shown in Figure 5.

\begin{tabular}{lcc}
\hline Coefficient & Figure 1(a) & Figure 1(b) \\
\hline$\widetilde{A}$ & $\frac{1}{2} k A^{2}\left|H_{1}\left(\omega_{o}\right)\right|\left|H_{2 a}\left(\omega_{o}\right)\right|$ & $\frac{1}{2} k A^{2}\left|H_{1}\left(\omega_{o}\right)\right|\left|H_{2 b}\left(\omega_{o}\right)\right|$ \\
$\widetilde{B}$ & $k A\left|H_{1}\left(\omega_{o}\right)\right| V_{\mathrm{dc}_{2}}$ & $\alpha k A\left|H_{1}\left(\omega_{o}\right)\right| V_{\mathrm{dc}_{1}}$ \\
$\widetilde{C}$ & $k A\left|H_{2 a}\left(\omega_{o}\right)\right|\left(V_{\mathrm{dc}_{1}}-V_{T}\right)$ & $k A\left|H_{2 b}\left(\omega_{o}\right)\right|\left[V_{\mathrm{dc}_{1}}-V_{T}\right]$ \\
$\widetilde{I}_{\text {off }}$ & $k\left[\left(V_{\mathrm{dc}_{1}}-V_{T}\right) V_{\mathrm{dc}_{2}}\right]$ & $\alpha k\left[V_{\mathrm{dc}_{1}}-V_{T}\right] V_{\mathrm{dc}_{1}}$ \\
\hline
\end{tabular}

$\widetilde{I}_{\text {off }}>\widehat{I}_{\text {off }}$, with all other terms in the coefficients being equal. The corresponding new pinch point $\left[\widetilde{V}_{\mathrm{in}_{p}}, \widetilde{I}_{\mathrm{in}_{p}}\right]$ is given by

$$
\begin{aligned}
\widetilde{V}_{\mathrm{in}_{p}}= & V_{\mathrm{dc}_{1}}-A \widetilde{X}(\Theta), \\
\widetilde{I}_{\mathrm{in}_{p}=} & -\widetilde{A} \cos \left[2 \sin ^{-1}(\widetilde{X}(\Theta))+\theta_{2 a, 2 b}-\theta_{1}\right] \\
& -\widetilde{B} \sin \left[\sin ^{-1}(\widetilde{X}(\Theta))-\theta_{1}\right] \\
& -\widetilde{C} \sin \left[\sin ^{-1}(\widetilde{X}(\Theta))+\theta_{2 a, 2 b}\right] \\
& +\widetilde{A} \cos \left(\theta_{1}+\theta_{2 a, 2 b}\right)+\widetilde{I}_{\text {off }},
\end{aligned}
$$

where

$$
\widetilde{X}(\Theta)=\frac{(\widetilde{B} / 2) \sin \left(-\theta_{1}\right)+(\widetilde{C} / 2) \sin \left(\theta_{2 a, 2 b}\right)}{\widetilde{A} \sin \left(\theta_{2 a, 2 b}-\theta_{1}\right)} .
$$

The benefits to linearizing $M_{1}$ are immediately clear upon close inspection of (31)-(33). In particular, for the circuit of Figure $1(\mathrm{a})$ in the general case when $\tau_{2}=\beta \tau_{1}$, the general solution to $\widetilde{X}(\Theta)=0$ now yields

$$
V_{\mathrm{dc}_{2}}=\frac{\beta\left(\tau_{1}^{2} \omega^{2}+1\right)}{\left(\beta^{2} \tau_{1}^{2} \omega^{2}+1\right)}\left(V_{\mathrm{dc}_{1}}-V_{T}\right),
$$

which, for $\beta=1$, implies that, for $\widetilde{V}_{\mathrm{in}_{p}}=V_{\mathrm{dc}_{1}}, V_{\mathrm{dc}_{2}}$ is chosen such that $V_{\mathrm{dc}_{2}}=V_{\mathrm{dc}_{1}}-V_{T}$. Of course, under these conditions, the composite linearized resistor is at the edge of the triode and a more practical solution would be for a given $\beta \neq 1$, $\omega$, and $\tau_{1}$ to simply choose $\beta\left(\tau_{1}^{2} \omega^{2}+1\right) /\left(\beta^{2} \tau_{1}^{2} \omega^{2}+1\right)>$ $V_{\mathrm{dc}_{2}} /\left(V_{\mathrm{dc}_{1}}-V_{T}\right)$. For the circuit of Figure 1(b) employing a linearized $M_{1}$, the choice of $\alpha$ does not affect the pinch point; however, the value of $V_{\mathrm{dc}_{1}}$ that results in $\widetilde{V}_{\mathrm{in}_{p}}=V_{\mathrm{dc}_{1}}$ is given by

$$
\begin{aligned}
& V_{\mathrm{dc}_{1}} \\
& =\frac{V_{T}}{1-\omega \tau_{1} \csc \left(\theta_{2 b}\right) \sqrt{\left(1+\tau_{p}^{2} \omega^{2}\right) /\left(1+\tau_{z}^{2} \omega^{2}\right)\left(1+\tau_{1}^{2} \omega^{2}\right)}},
\end{aligned}
$$

which is still frequency dependent, but minimization is still possible if $\omega \tau_{1}>1, \omega \tau_{z}>1$, and $\omega \tau_{p}>1$. Last but not least, no pinching occurs for the circuit of Figure 1(a) using the linearized resistor at frequencies

$$
\begin{aligned}
& \omega_{1}=\frac{1}{\tau_{1}} \sqrt{\frac{V_{\mathrm{dc}_{2}}+V_{T}}{2 V_{\mathrm{dc}_{1}}-V_{\mathrm{dc}_{2}}-V_{T}},} \\
& \omega_{2}=\frac{1}{\tau_{1}} \sqrt{\frac{2 V_{\mathrm{dc}_{1}}-V_{\mathrm{dc}_{2}}-V_{T}}{V_{\mathrm{dc}_{1}}+2 V_{\mathrm{dc}_{2}}+V_{T}}}
\end{aligned}
$$

assuming $\tau_{2}=\tau_{1}$ and at

$$
\omega_{1}=\sqrt{\frac{2 V_{\mathrm{dc}_{1}}-V_{T}}{V_{\mathrm{dc}_{1}} \tau_{1} \tau_{p}-\tau_{1}^{2}\left(V_{\mathrm{dc}_{1}}-V_{T}\right)}},
$$

$$
\omega_{2}=\sqrt{\frac{\left(2 V_{\mathrm{dc}_{1}}-V_{T}\right) \tau_{p}+V_{T} \tau_{1}}{\left(\tau_{1}^{3}-2 \tau_{1}^{2} \tau_{p}-\tau_{p}^{2}\right) V_{\mathrm{dc}_{1}}+\left(\tau_{p}-\tau_{1}\right) \tau_{1}^{2} V_{T}}},
$$

for the circuit of Figure 1(b) when $\tau_{z}=\tau_{1}=\alpha \tau_{p}$.

\section{Simulation and Experimental Results}

The circuits in Figures 1(a) and 1(b) were simulated and built experimentally. In the sections that follow, simulations of Figures 1(a) and 1(b) without and with linearization of $M_{1}$ were conducted. For the experiments, off-the-shelf discrete components were used without linearization of $M_{1}$.

5.1. Simulation Results: Without $M_{1}$ Linearization. For simulation purposes, Cadence was used employing the Design Kit offered by the AMS $0.35 \mu \mathrm{m}$ CMOS process. The opamp utilized in simulations is demonstrated in Figure 6, where the bias scheme was $V_{\mathrm{DD}}=-V_{\mathrm{SS}}=5 \mathrm{~V}$ and $I o=300 \mu \mathrm{A}$. The MOS transistors' aspect ratio is given in Table 4 with $R_{c}=$ $140 \Omega$ and $C_{c}=2 \mathrm{pF}$ to achieve a phase margin of $60^{\circ}$. Also, the aspect ratio of transistor $M_{1}$ in Figure 1(a) was $100 \mu \mathrm{m} / 1 \mu \mathrm{m}$; for $M_{2}$, the aspect ratio was $12 \mu \mathrm{m} / 2 \mu \mathrm{m}$ and thus its gain factor was $k=1 \mathrm{~mA} / \mathrm{V}^{2}$.

The resistor and capacitor values used in simulations were $R_{1}=820 \Omega$ and $C_{1}=C_{2}=470 \mathrm{nF}$ and, therefore, $\tau_{1}=$ $\tau_{2}$. The DC voltages were $V_{\mathrm{dc}_{1}}=2 \mathrm{~V}$ and $V_{\mathrm{dc}_{2}}=0.74 \mathrm{~V}$. Considering a sinusoidal input with $700 \mathrm{mV}$ amplitude and variable frequency, the obtained $i_{\text {in }_{a}}-v_{\text {in }}$ characteristics, for $f=600 \mathrm{~Hz}, 1 \mathrm{kHz}$, and $1.5 \mathrm{kHz}$, are demonstrated in Figure 7 . The time-domain behavior of the scheme in Figure 1(a) is demonstrated in Figure 8 for a $1 \mathrm{kHz}$ input voltage. Likewise, 


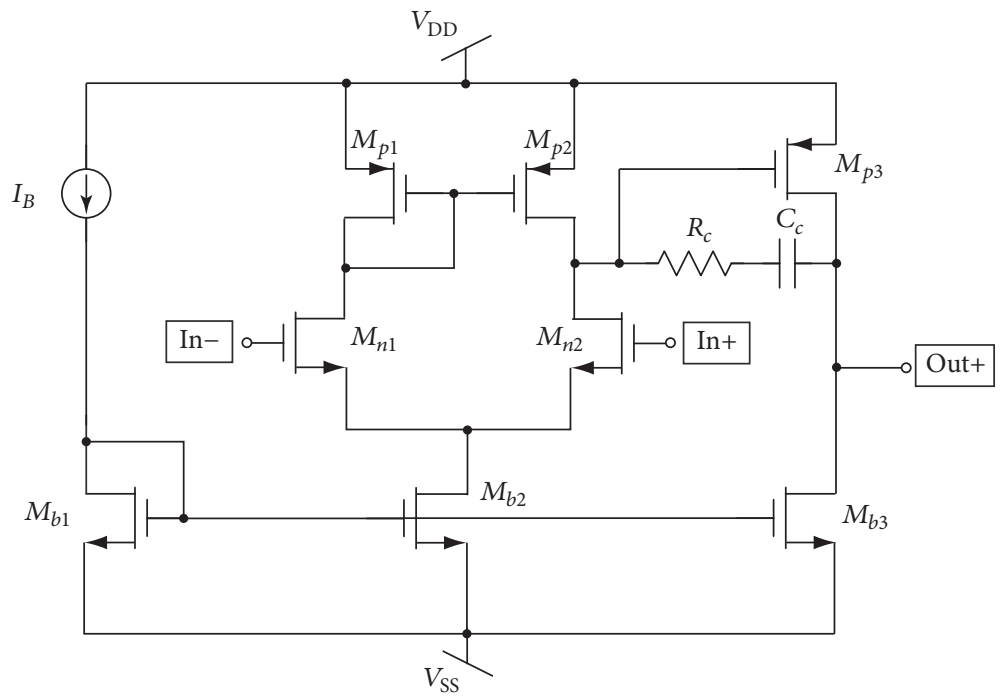

FIGURE 6: Opamp used in simulations.

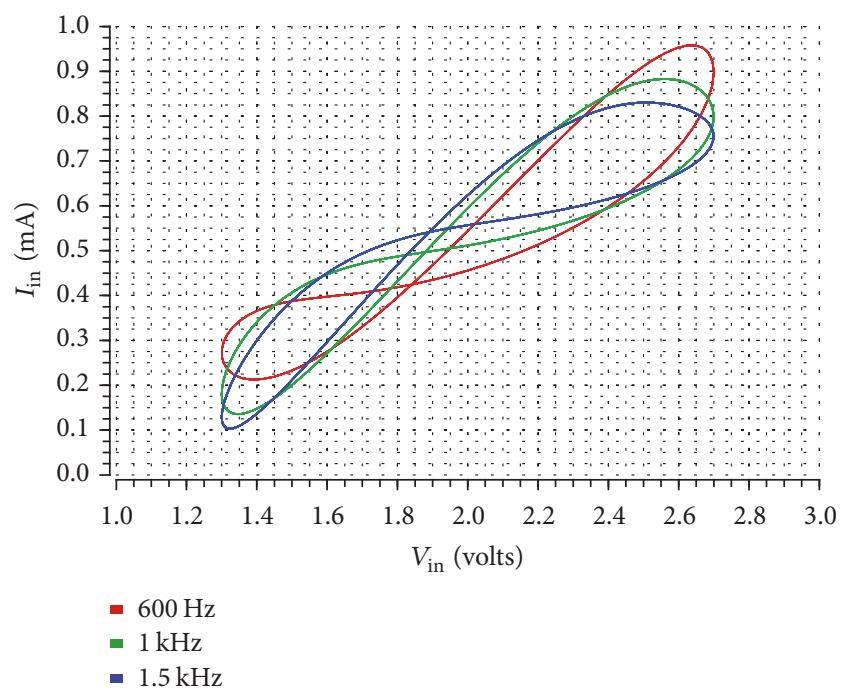

FIgURE 7: Simulated $i_{\mathrm{in}_{a}}-v_{\text {in }}$ characteristics for $f=600 \mathrm{~Hz}, 1 \mathrm{kHz}$, and $1.5 \mathrm{kHz}$.

TABLE 4: MOS transistors' aspect ratio for Figure 6.

\begin{tabular}{lc}
\hline Transistor & $W / L$ \\
\hline$M_{b 1}$ & $50 \mu \mathrm{m} / 2 \mu \mathrm{m}$ \\
$M_{b 2}-M_{b 3}$ & $100 \mu \mathrm{m} / 2 \mu \mathrm{m}$ \\
$M_{n 1}-M_{n 2}$ & $200 \mu \mathrm{m} / 0.5 \mu \mathrm{m}$ \\
$M_{p 1}-M_{p 2}$ & $50 \mu \mathrm{m} / 0.5 \mu \mathrm{m}$ \\
$M_{p 3}$ & $200 \mu \mathrm{m} / 0.5 \mu \mathrm{m}$ \\
\hline
\end{tabular}

the obtained $i_{\text {in }_{b}}-v_{\text {in }}$ characteristics, for Figure 1(b) for $f=$ $400 \mathrm{~Hz}, 700 \mathrm{~Hz}$, and $2 \mathrm{kHz}$, are demonstrated in Figure 9 with the corresponding time-domain behavior shown in Figure 10 for a $700 \mathrm{~Hz}$ input voltage.

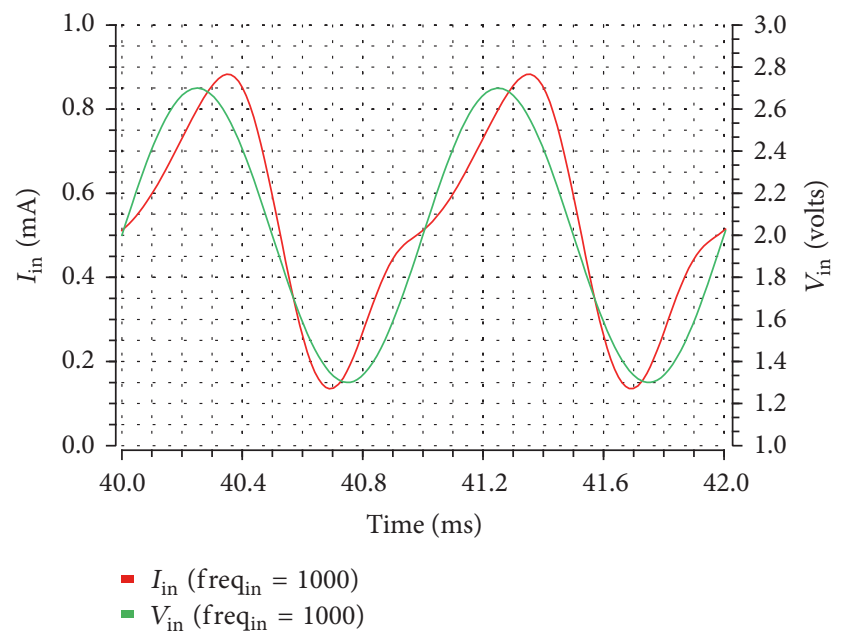

FIGURE 8: Time-domain behavior of the circuit in Figure 1(a) for $f=$ $1 \mathrm{kHz}$.

The effect of $r_{2}$ in the operation of the topology in Figure 1(b) has also been studied under the conditions $r_{2}=0.4 r_{1}, 0.56 r_{1}$, and $0.7 r_{1}$, which sets $\alpha=$ $0.26,0.36$, and 0.41 , respectively. The derived $i_{\text {in }_{b}}-v_{\text {in }}$ characteristics, for $f=700 \mathrm{~Hz}$, are given in Figure 11 .

5.2. Simulation Results: With $M_{1}$ Linearization. The improved linear resistor shown in Figure 5 was also used for linearizing transistor $M_{1}$. The power supply voltage was equal to $2 \mathrm{~V}$ and the aspect ratio of $M_{1}-M_{2}$ and $M_{5}-M_{6}$ was $1 \mu \mathrm{m} / 2 \mu \mathrm{m}$, while for $M_{3}-M_{4}$ it was $3.2 \mu \mathrm{m} / 2 \mu \mathrm{m}$. Considering a sinusoidal input with $700 \mathrm{mV}$ amplitude and variable frequency, the obtained $i_{\text {in }_{a}}-v_{\text {in }}$ characteristics, for the circuit in Figure 1(a), derived at the same conditions as in the previous subsection, and for $f=600 \mathrm{~Hz}, 1 \mathrm{kHz}$, and $1.5 \mathrm{kHz}$, are demonstrated in Figure 12. The corresponding 


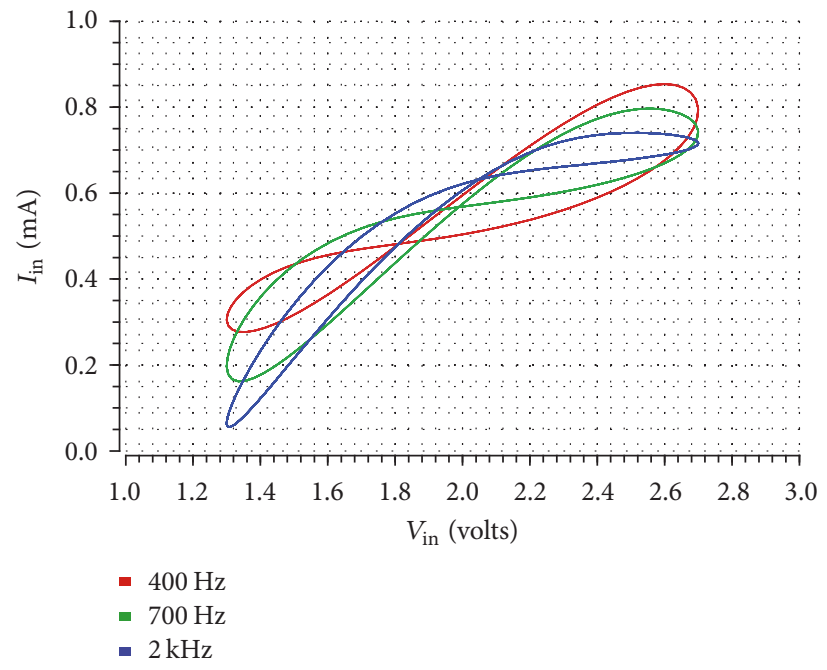

FIGURE 9: Simulated $i_{\mathrm{in}_{b}}-v_{\text {in }}$ characteristics for $f=400 \mathrm{~Hz}, 700 \mathrm{~Hz}$, and $2 \mathrm{kHz}$.

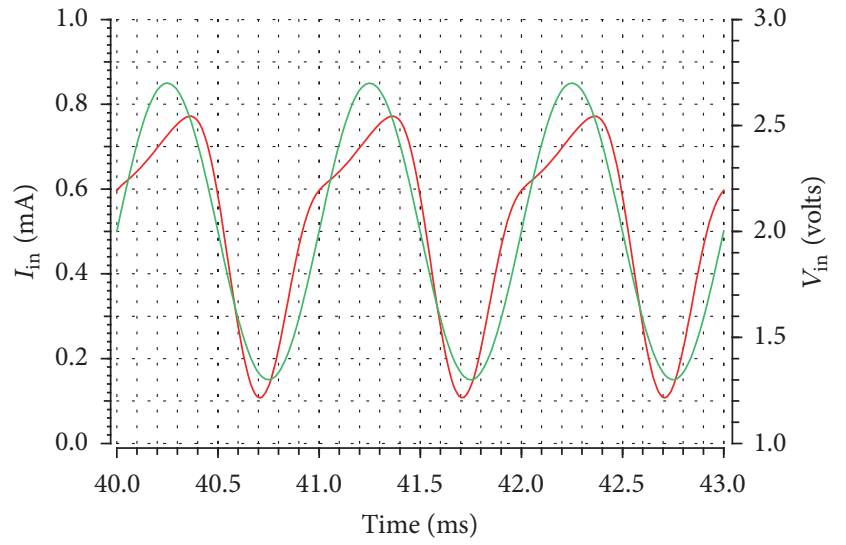

- $I_{\text {in }}$

Figure 10: Time-domain behavior of the circuit in Figure 1(b) for $f=700 \mathrm{~Hz}$.

time-domain behavior is depicted in Figure 13 for a $1 \mathrm{kHz}$ input voltage.

In a similar way, the plots for the circuit in Figure 1(b), obtained at the same conditions as in the previous subsection, are given in Figure 14. The time-domain behavior is given in Figure 15 for a $700 \mathrm{~Hz}$ input voltage.

5.3. Experimental Results. In the first of a series of experimental tests, the circuit of Figure 1(a) was constructed using 741 opamps powered by a $\pm 15 \mathrm{~V}$ supply. The resistor and capacitor values used were $R_{1}=R_{2}=820 \Omega$ and $C_{1}=$ $C_{2}=470 \mathrm{nF}$ ensuring that $\tau_{1}=\tau_{2}$. Transistors $M_{1}$ and $M_{2}$ were taken from Fairchild's dual complementary pair CD 4007CN chip. The DC biasing voltages used were $V_{\mathrm{dc}_{1}}=$ $4.6 \mathrm{~V}, V_{\mathrm{dc}_{2}}=1.12 \mathrm{~V}$, and $=1 \mathrm{~V}_{\mathrm{pp}}$. The current $i_{\mathrm{in}_{a}}$ was measured by inserting a $10 \Omega$ resistor in series with $v_{\text {in }}$ and measuring the voltage drop across this resistor using an

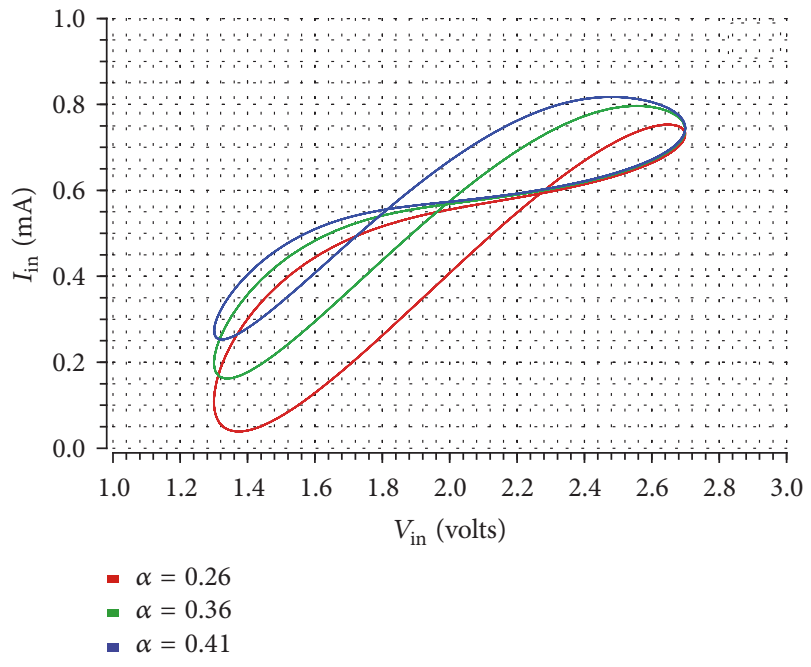

FIGURE 11: Simulated $i_{\mathrm{in}_{b}}-v_{\text {in }}$ characteristics for $\alpha=0.26,0.36$, and 0.41 .

instrumentation amplifier with a gain of 10; that is, $i_{\mathrm{in}_{a}}=v_{10 \Omega}$. The results of $v_{\text {in }}$ versus $i_{\text {in }_{a}}$ are shown in Figure 16 for several frequencies starting at $1 \mathrm{kHz}$ with the pinch point remaining nearly constant for $V_{\mathrm{dc}_{2}}=1.12 \mathrm{~V}$. Note that this result is consistent with (22) where SPICE models for the CD4007 (the actual value of $V_{T}$ is both foundry and process dependent but unfortunately actual data on the CD4007CN chips used was not available) place $V_{T}$ in the order of around $2 \sim 2.3 \mathrm{~V}$. Bending of the lobes can be observed towards a downward trend when the frequency is decreased below $1 \mathrm{kHz}$ and upwards when the frequency is increased above $1 \mathrm{kHz}$. The usable range of this circuit was found to be from $300 \mathrm{~Hz}$ to $10 \mathrm{kHz}$. Note that the lower frequency limit on the operation of the circuit $(300 \mathrm{~Hz})$ is also consistent with (25) where, for $V_{\mathrm{dc}_{2}}=(1 / 2)\left(V_{\mathrm{dc}_{1}}-V_{T}\right), R_{1}=820 \Omega, C_{1}=470 \mathrm{nF}$, with $\tau_{1}=\tau_{2}$, yields a calculated value of $\omega_{1,2}=292 \mathrm{~Hz}$. The upper frequency limit was observed when the lobes 


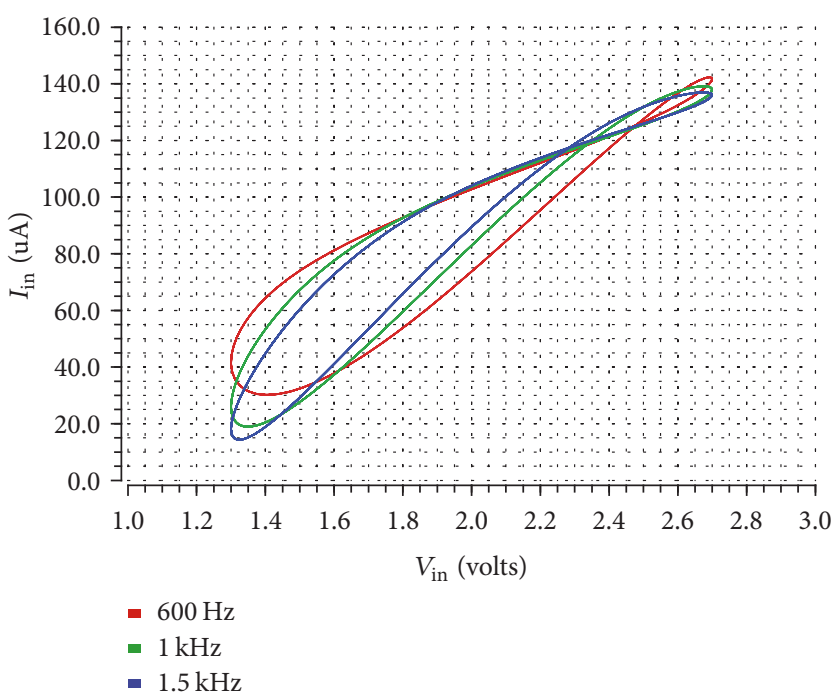

FIGURE 12: Simulated $i_{\mathrm{in}_{a}}-v_{\text {in }}$ characteristics for the circuit in Figure 1 (a) with $f=600 \mathrm{~Hz}, 1 \mathrm{kHz}$, and $1.5 \mathrm{kHz}$ and linearization of $M_{1}$.

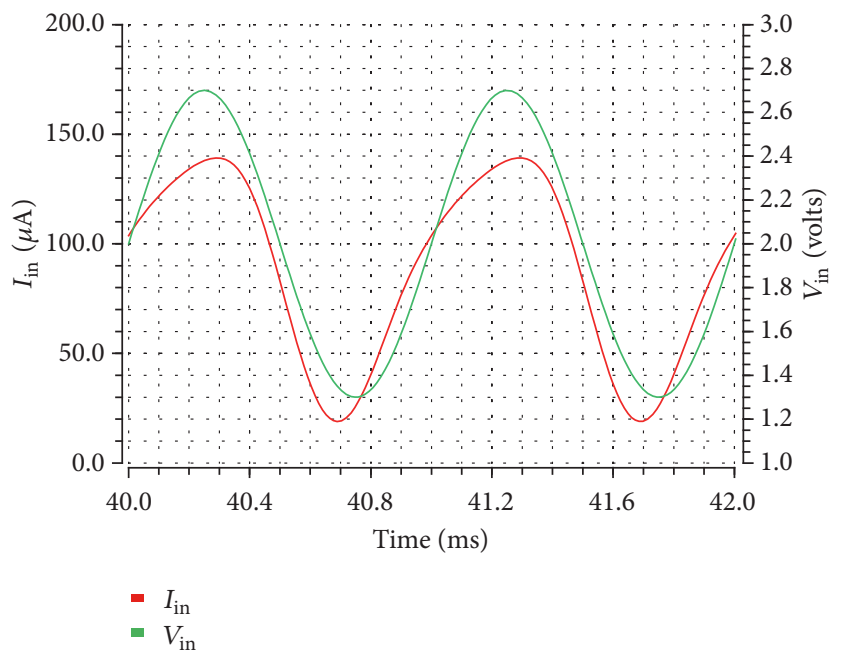

FIGURE 13: Time-domain behavior of the circuit in Figure 1(a) for $f=1 \mathrm{kHz}$ and linearization of $M_{1}$.

of the pinch hysteresis were too close to be distinguishable and in addition would also be set by limitations associated with $A_{2}$ losing gain in its closed-loop configuration with $M_{2}$.

In a second experimental test, the circuit of Figure 1(b) was set up and the resistor and capacitor values used were $R_{1}=r_{2}=2 \mathrm{k} \Omega$ and $C_{1}=C_{2}=470 \mathrm{nF}$ ensuring that $\tau_{z}=\tau_{1}$. The current $i_{\mathrm{in}_{b}}$ was likewise measured through a $10 \Omega$ resistor using an instrumentation amplifier set to a gain of 10 . Resistor $r_{1}$ was adjusted by a potentiometer at a value of $r_{1}=1031 \Omega$ which set $\alpha=0.34$. Input voltages were set at $V_{\mathrm{dc}_{1}}=4 \mathrm{~V}$ and $A=3 \mathrm{~V}_{\mathrm{pp}}$ and the initial frequency was set at $700 \mathrm{~Hz}$. The results shown in Figure 17 indicate that the pinch point and symmetry of the lobes are highly dependent on the input frequency. For this configuration, pinching was lost for frequencies below $300 \mathrm{~Hz}$ and above $10 \mathrm{kHz}$.

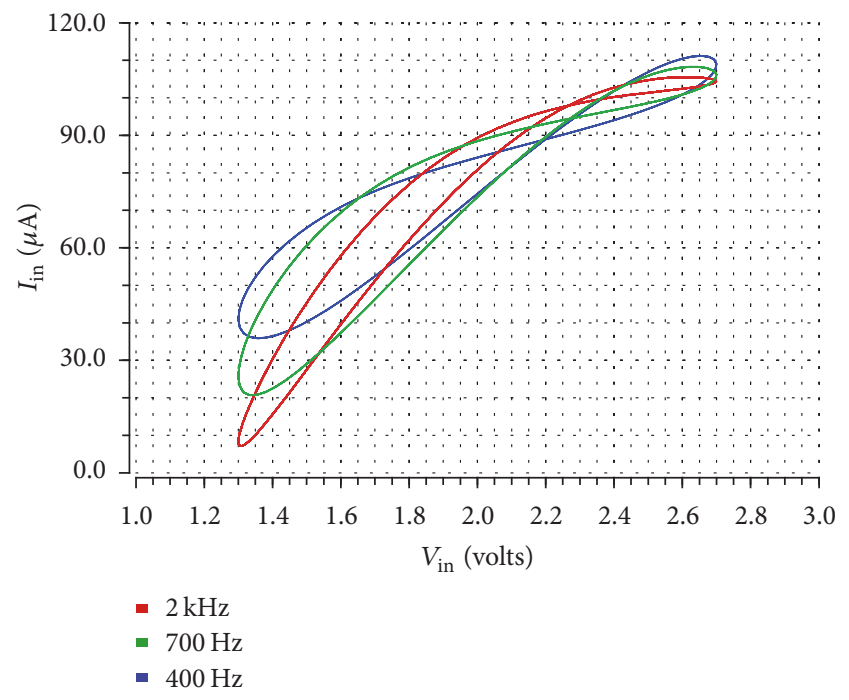

FIGURE 14: Simulated $i_{\operatorname{in}_{b}}-v_{i n}$ characteristics for the circuit in Figure 1(b) with $f=400 \mathrm{~Hz}, 700 \mathrm{~Hz}$, and $2 \mathrm{kHz}$ and linearization of $M_{1}$.

Finally, it should be mentioned that the circuits of Figures 1(a) and 1(b) were also tested using different time constants such as $\tau_{1} \neq \tau_{2}$ and $\tau_{1} \neq \tau_{z}$ and all results not shown here were consistent with the expected theory.

\section{Conclusion}

Two simple nonlinear circuits that exhibit unipolar pinched hysteresis behavior were presented in this paper. The multiplication-type nonlinearity between a state variable and its past history, as given in (1), is fundamental in obtaining pinched hysteresis although the past history can also be replaced by the rate of change of the present state as shown in [3]. In this work, this multiplication is simply achieved 


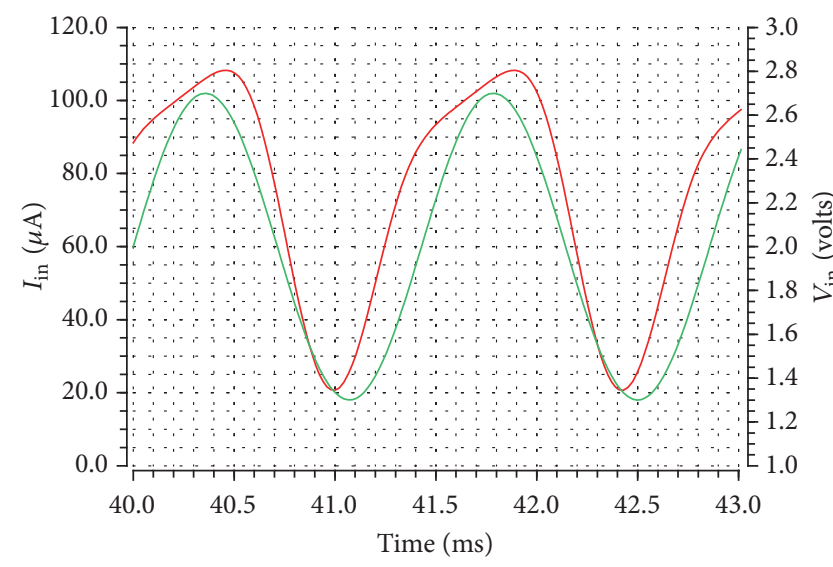

- $I_{\text {in }}$

- $V_{\text {in }}$

FIgURE 15: Time-domain behavior of the circuit in Figure 1(b) for $f=700 \mathrm{~Hz}$ and linearization of $M_{1}$.

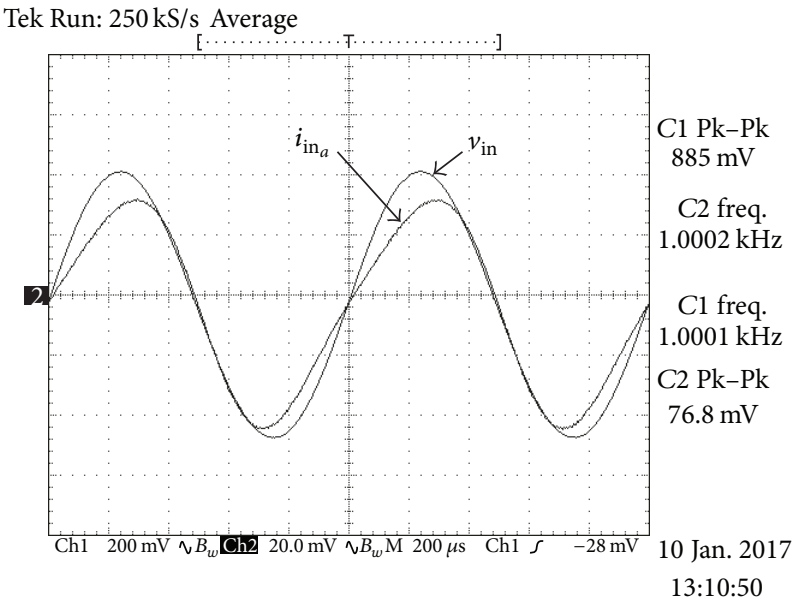

(a)

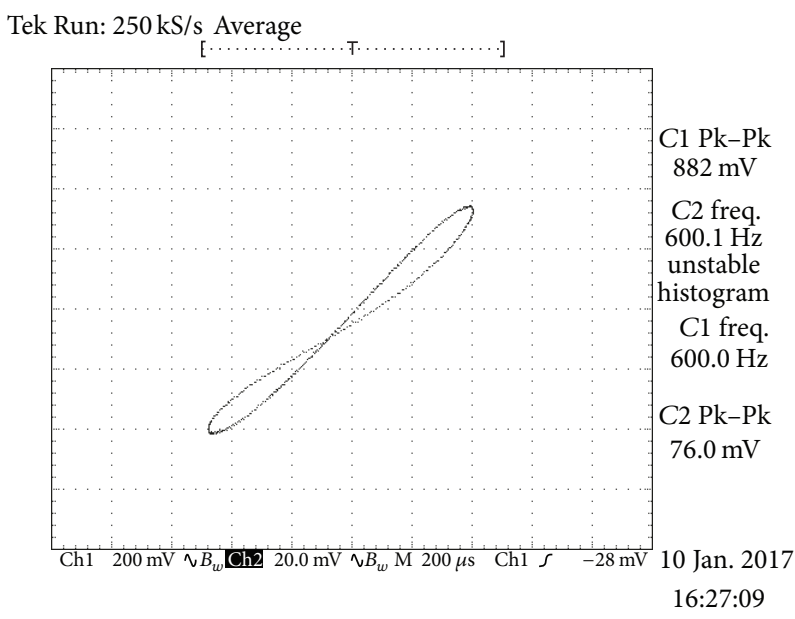

(c)

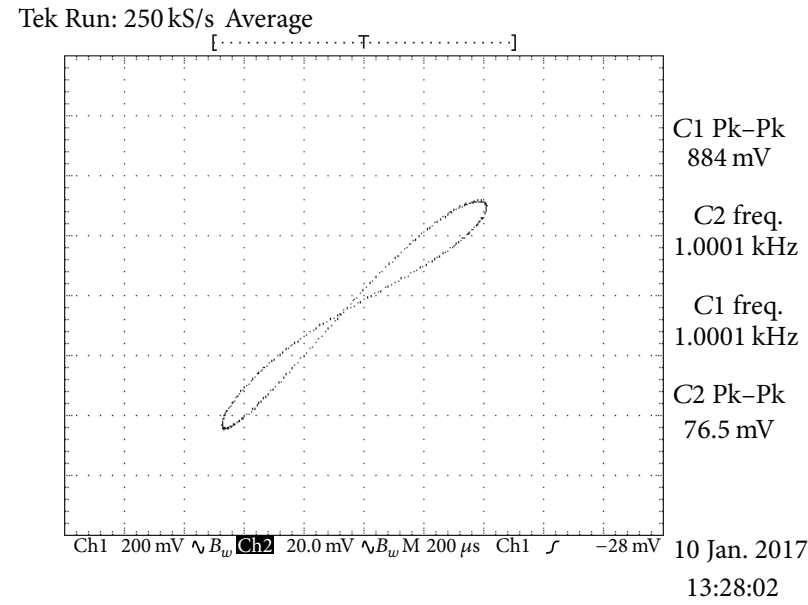

(b)

Tek Run: $250 \mathrm{kS} / \mathrm{s}$ Average

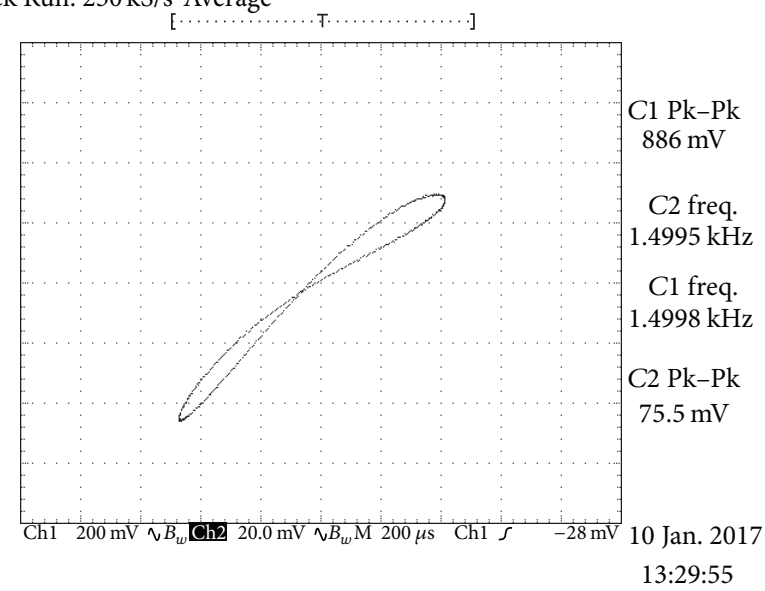

(d)

FIGURE 16: (a) Experimental results of the plot of $v_{\text {in }}(t)$ and $i_{\text {in }}(t)$ at $1 \mathrm{kHz}$ for the circuit of Figure 1(a). (b) Oscilloscope trace of the pinched hysteresis loop of the memristor emulator circuit of Figure $1(\mathrm{a})$ at $1 \mathrm{kHz}$, (c) at $600 \mathrm{~Hz}$, and (d) at $1.5 \mathrm{kHz}$. 


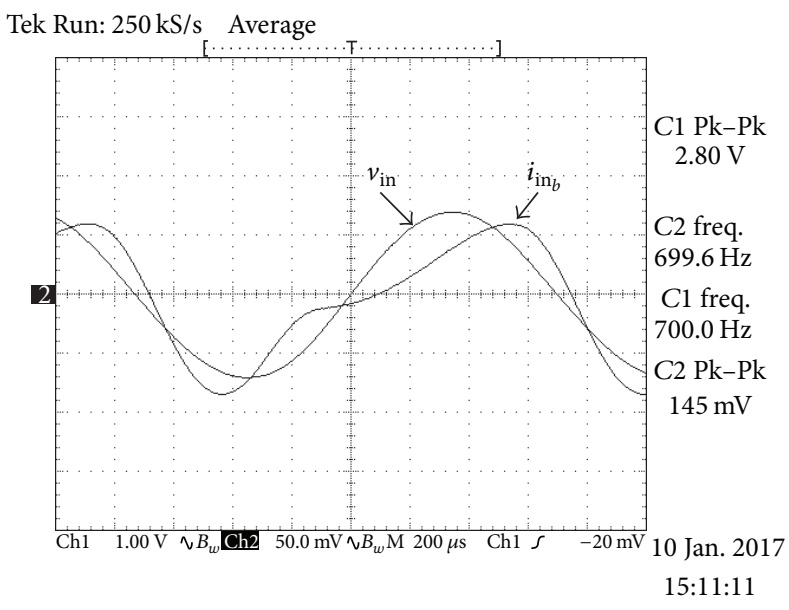

(a)

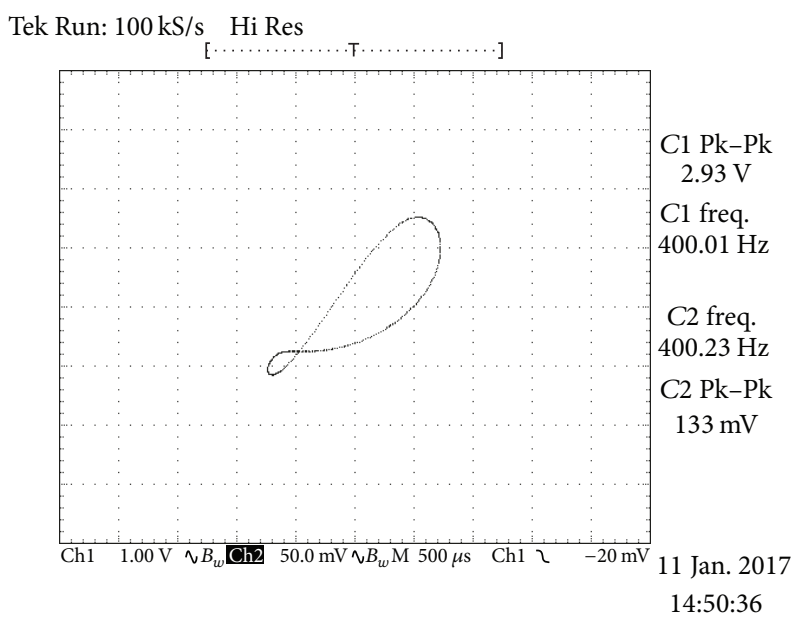

(c)

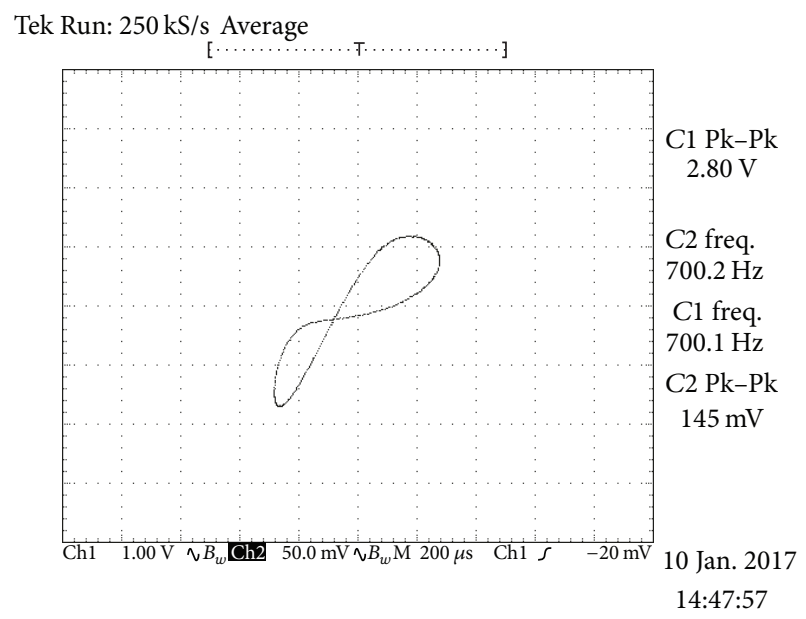

(b)

Tek Run: 500 kS/s Hi Res

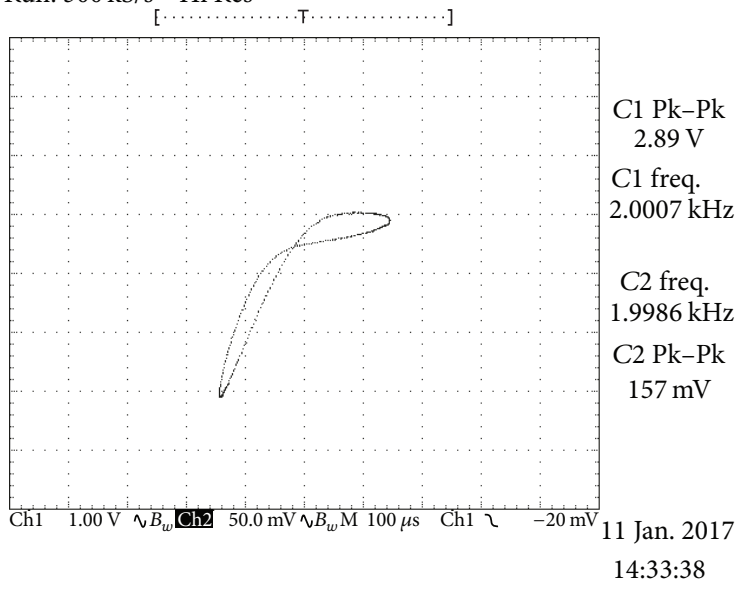

(d)

FIGURE 17: (a) Experimental results of the plot of $v_{\mathrm{in}}(t)$ and $i_{\mathrm{in}_{b}}(t)$ at $700 \mathrm{~Hz}$ for the circuit of Figure 1(b). (b) Oscilloscope trace of the pinched hysteresis loop at $700 \mathrm{~Hz},(\mathrm{c})$ at $400 \mathrm{~Hz}$, and (d) at $2 \mathrm{kHz}$.

using the MOS transistor transconductance equation. The proposed circuits have been analyzed, their pinch points were determined, and their behavior was verified in Matlab and experimentally. A method of linearization that enables the elimination of undesired higher-order nonlinear terms was also examined and verified via simulations in Cadence. Of significant importance in this work is the clarification of the role played by the charge holding capacitor in the value of the charge-controlled memristance. We argue that, in all solid-state devices that have been fabricated and that show pinched hysteresis, a parasitic capacitor combined with a modulation-type (multiplication-type) nonlinearity is behind the appearance of this behavior. Arguably, the authors of [18] conclude that both "memory resistance and memory capacitance must coexist."

\section{Conflicts of Interest}

The authors declare that they have no conflicts of interest.

\section{Acknowledgments}

The authors would like to acknowledge the support of the Natural Sciences and Engineering Research Council (NSERC) of Canada in this work and Mr. Abdulwadood AlAli in the preparation and the carrying out of the experiments.

\section{References}

[1] Z. Biolek, D. Biolek, and V. Biolková, "Specification of one classical fingerprint of ideal memristor," Microelectronics Journal, vol. 46, no. 4, pp. 298-300, 2015.

[2] F. Corinto, P. P. Civalleri, and L. O. Chua, "A theoretical approach to memristor devices," IEEE Journal on Emerging and Selected Topics in Circuits and Systems, vol. 5, no. 2, pp. 123-132, 2015.

[3] M. E. Fouda, A. S. Elwakil, and A. G. Radwan, "Pinched hysteresis with inverse-memristor frequency characteristics in some nonlinear circuit elements," Microelectronics Journal, vol. 46, no. 9, article no. 3861, pp. 834-838, 2015. 
[4] A. Ascoli, R. Tetzlaff, Z. Biolek, Z. Kolka, V. Biolkova, and D. Biolek, "The art of finding accurate memristor model solutions," IEEE Journal on Emerging and Selected Topics in Circuits and Systems, vol. 5, no. 2, pp. 133-142, 2015.

[5] S. Vongehr and X. Meng, "The missing memristor has not been found,” Scientific Reports, vol. 5, Article ID 11657, 2015.

[6] A. S. Elwakil, M. E. Fouda, and A. G. Radwan, "A simple model of double-loop hysteresis behavior in memristive elements," IEEE Transactions on Circuits and Systems II: Express Briefs, vol. 60, no. 8, pp. 487-491, 2013.

[7] M. T. Abuelma'Atti and Z. J. Khalifa, "A continuous-level memristor emulator and its application in a multivibrator circuit," AEU - International Journal of Electronics and Communications, vol. 69, no. 4, pp. 771-775, 2015.

[8] C. Sánchez-López, M. A. Carrasco-Aguilar, and C. MuñizMontero, "A $16 \mathrm{~Hz}-160 \mathrm{kHz}$ memristor emulator circuit," AEU - International Journal of Electronics and Communications, vol. 69, no. 9, pp. 1208-1219, 2015.

[9] A. G. Alharbi, Z. J. Khalifa, M. E. Fouda, and M. H. Chowdhury, "Memristor emulator based on single CCII," in Proceedings of the 27th International Conference on Microelectronics, ICM 2015, pp. 174-177, IEEE, Casablanca, Morocco, December 2015.

[10] C. Yang, S. Cho, M. P. Sah, H. Kim, and K.-S. Jung, "Memristor emulator with off-the-shelf solid state components for memristor application circuits," in Proceedings of the 2012 13th International Workshop on Cellular Nanoscale Networks and their Applications, CNNA 2012, IEEE, Piscataway, NJ, USA, August 2012.

[11] A. I. Hussein and M. E. Fouda, "A simple MOS realization of current controlled memristor emulator," in Proceedings of the 2013 25th International Conference on Microelectronics, ICM 2013, IEEE, Beirut, Lebanon, December 2013.

[12] A. Elwakil, B. Maundy, and C. Psychalinos, "On the pinched hysteresis behavior in a state-controlled resistor," AEU - International Journal of Electronics and Communications, vol. 74, pp. 171-175, 2017.

[13] R. Mutlu and E. Karakulak, "Emulator circuit of $\mathrm{Ti}_{2}$ memristor with linear dopant drift made using analog multiplier," in Proceedings of the 2010 7th National Conference on Electrical, Electronics and Computer Engineering, ELECO 2010, pp. 380384, Bursa, Turkey, December 2010.

[14] A. G. Alharbi, M. E. Fouda, Z. J. Khalifa, and M. H. Chowdhury, "Simple generic memristor emulator for voltage-controlled models," in Proceedings of the 2016 IEEE 59th International Midwest Symposium on Circuits and Systems (MWSCAS), pp. 14, Abu Dhabi, United Arab Emirates, October 2016.

[15] Y. Babacan and F. Kaçar, "Floating memristor emulator with subthreshold region," Analog Integrated Circuits and Signal Processing, vol. 90, no. 2, pp. 471-475, 2017.

[16] M. T. Abuelma'atti and Z. J. Khalifa, "A new floating memristor emulator and its application in frequency-to-voltage conversion," Analog Integrated Circuits and Signal Processing, vol. 86, no. 1, pp. 141-147, 2016.

[17] Z. B. Yan and J.-M. Liu, "Coexistence of high performance resistance and capacitance memory based on multilayered metal-oxide structures," Scientific Reports, vol. 3, article no. 2482, 2013.

[18] I. Salaoru, Q. Li, A. Khiat, and T. Prodromakis, "Coexistence of memory resistance and memory capacitance in $\mathrm{TiO} 2$ solid-state devices," Nanoscale Research Letters, vol. 9, no. 1, pp. 1-7, 2014.

[19] N. Tadić and D. Gobović, "A voltage-controlled resistor in CMOS technology using bisection of the voltage range," IEEE
Transactions on Instrumentation and Measurement, vol. 50, no. 6, pp. 1704-1710, 2001.

[20] B. Maundy, S. Gift, and P. Aronhime, "Practical voltage/currentcontrolled grounded resistor with dynamic range extension," IET Circuits, Devices and Systems, vol. 2, no. 2, pp. 201-206, 2008 .

[21] M. Kushima, M. Inaba, and K. Tanno, "Linear and compact floating node voltage-controlled variable resistor circuit," IEICE Transactions on Fundamentals of Electronics, Communications and Computer Sciences, vol. E89-A, no. 2, pp. 459-460, 2006.

[22] B. Maundy and M. Maini, "A comparison of three multipliers based on the Vgs2 technique for low-voltage applications," IEEE Transactions on Circuits and Systems I: Fundamental Theory and Applications, vol. 50, no. 7, pp. 937-940, 2003. 


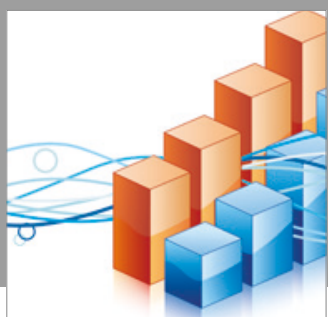

Advances in

Operations Research

vatersals

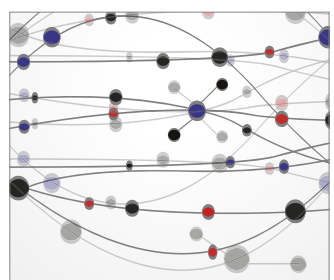

\section{The Scientific} World Journal
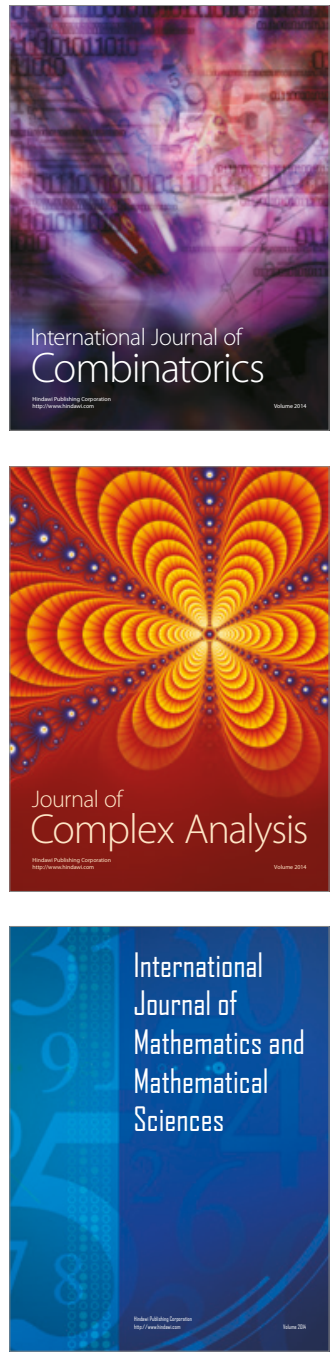
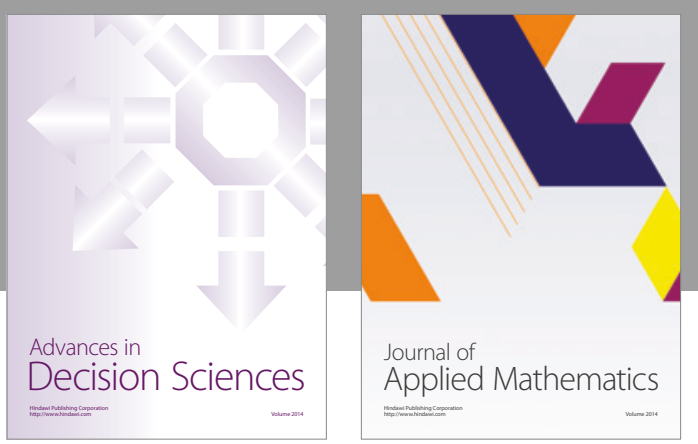

Algebra

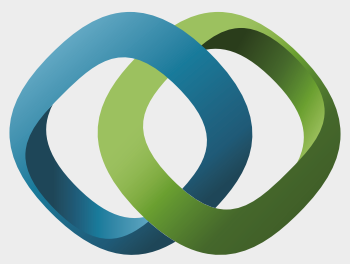

\section{Hindawi}

Submit your manuscripts at

https://www.hindawi.com
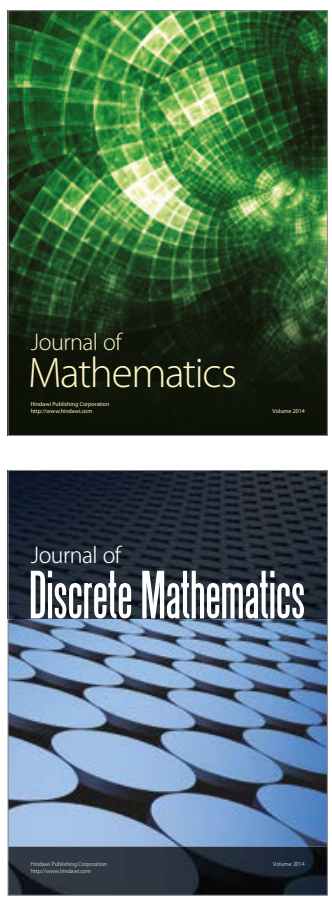

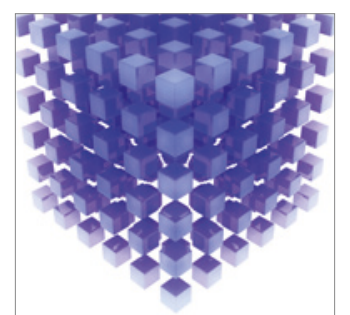

Mathematical Problems in Engineering
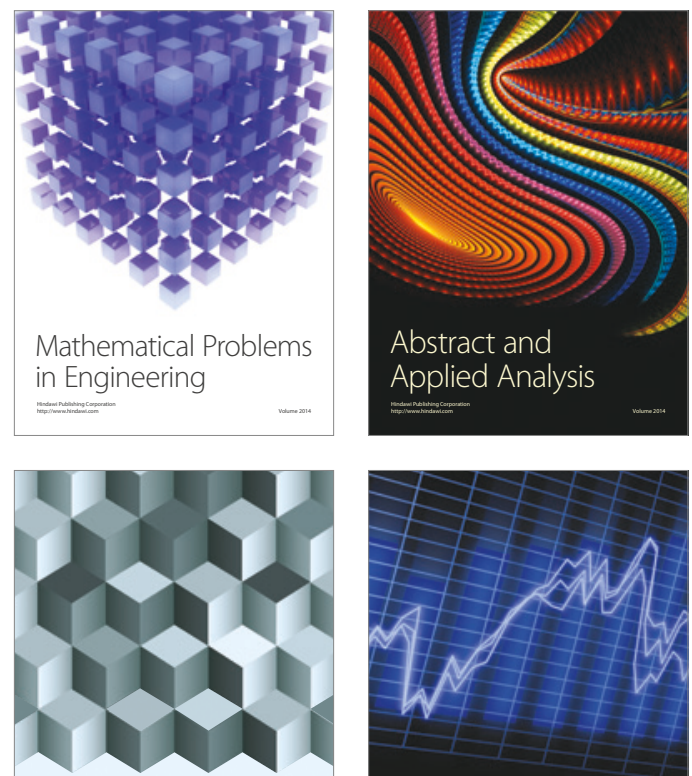

Journal of

Function Spaces

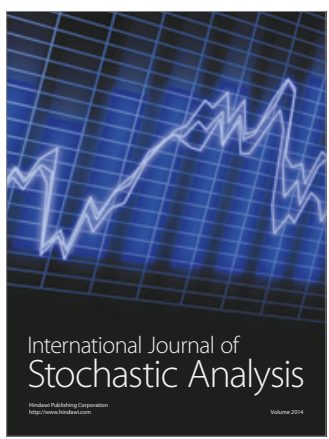

Probability and Statistics
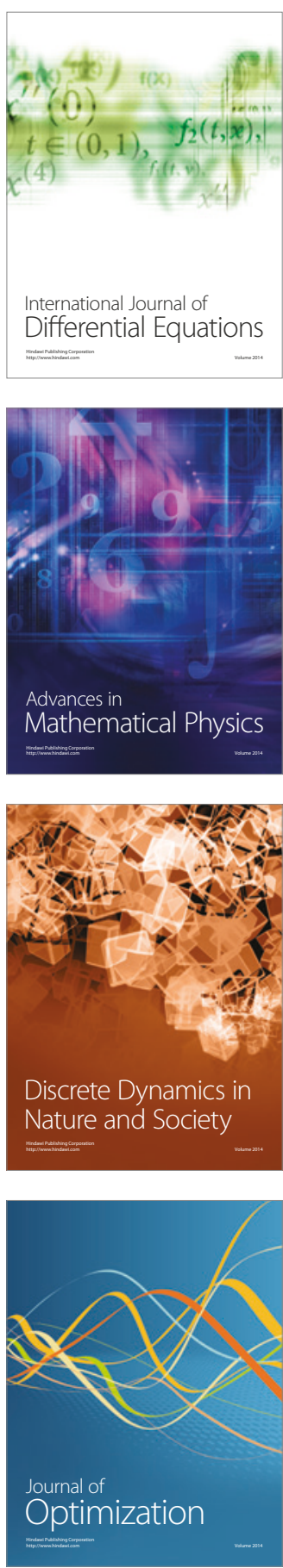